\title{
Macromolecular theory of solvation and structure in mixtures of colloids and polymers
}

\author{
M. Fuchs ${ }^{1, *}$ and K. S. Schweizer ${ }^{2}$ \\ ${ }^{1}$ Department of Physics and Astronomy, The University of Edinburgh, JCMB King's Buildings, Mayfield Road, \\ Edinburgh EH9 3JZ, United Kingdom \\ ${ }^{2}$ Departments of Materials Science and Engineering and Chemistry and Materials Research Laboratory, University of Illinois, \\ Urbana, Illinois 61801
}

(Received 8 February 2001; published 27 July 2001)

\begin{abstract}
The structural and thermodynamic properties of mixtures of colloidal spheres and nonadsorbing polymer chains are studied within a general two-component macromolecular liquid state approach applicable for all size asymmetry ratios. The dilute limits, when one of the components is at infinite dilution but the other concentrated, are presented and compared to field theory and to models that replace polymer coils with spheres. Whereas the derived analytical results compare well, qualitatively and quantitatively, with mean-field scaling laws where available, important differences from "effective sphere" approaches are found for large polymer sizes or semidilute concentrations.
\end{abstract}

PACS number(s): 61.20.-p, 82.70.Dd, 61.25.Hq

\section{INTRODUCTION}

Mixtures of dispersed spherical particles and nonadsorbing polymers may be viewed as a model system for a wide variety of materials encountered in food products, biological systems, or technological applications. In these systems, the "depletion attraction" is always present because it has a purely entropic and universal origin. Its consequences can most clearly be studied in mixtures of colloidal hard spheres and polymer chains made up of hard units where only the entropic consideration of the packing of particles restricted by steric or excluded volume constraints enters.

Because of the fundamental nature of the depletion attraction, it has been studied theoretically since the pioneering work of Asakura and Oosawa [1] and Vrij [2]. Moreover, its effect on the phase behavior had been observed much earlier [3]. The phase diagram of colloid-polymer mixtures has been constructed using the Asakura-Oosawa pair potential in an effective one-component thermodynamic perturbation calculation [4], within a two-component dilute polymer free volume approach [5], and also with computer simulations [6,7], the latter based on specific models originating in Refs. [1,2]. The Asakura-Oosawa model consists of replacing the polymer coils with effective spheres that can freely interpenetrate each other but not the colloidal spheres. This model has been further treated by liquid state theory [8], has been extended to nonhomogeneous situations $[9,10]$ and to perturbatively include polymer nonideality [11]. The forces it predicts for dilute and rather large colloidal spheres have been measured directly $[12,13]$ and phase diagrams for colloidal spheres appreciably larger than the polymers have been obtained, and agree semiquantitatively with theory [14-16].

More detailed experiments on the colloidal correlations [6,17-19], measurements of the second virial coefficient [20], and quantitative tests of the phase diagrams for larger polymer sizes $[15,18,19,21-23]$ have, however, detected

\footnotetext{
*Permanent address: Physik-Department, Technische Universität München, 85747 Garching, Germany.
}

polymer correlations that are not contained in the mentioned approaches. Also, for small spherical surfactant micelles [24] the dependence of phase separation on polymer size is opposite to predictions of colloid approaches [4,5]. There are at least two reasons for these discrepancies. First, polymer coils can deform close to particles and can thus fit into void spaces more effectively than spheres. Second, for higher polymer concentrations the coils start to overlap and the relevant polymer correlation length crosses over from the coil radius to the size of a mesh in the formed transient network. Both effects are important if the polymer coils are not negligibly small compared to the particles and both are neglected in the described theoretical approaches. The effects have long been understood from field-theoretic approaches to polymers in the limit of dilute colloidal particles. The deformability of the polymer coils affects the depletion layer of polymer segments close to particles, the resulting insertion free energies, and the induced colloid pair interactions [25-29]. For semidilute polymer suspensions the depletion layer and the induced interactions were obtained for both large and small colloids [27-33]. Yet, except for in a highly idealized meanfield thermodynamic perturbation calculation of hard spheres by Schaink and Smit [34], polymer field-theoretical approaches have not been extended to finite colloid concentrations.

Recently we proposed a macromolecular liquid state theory for mixtures of arbitrary polymer to colloid size ratios [35], which, although it is not rigorous for dilute systems, presents a viable and first principles approach for finite densities. It is unique in its applicability to all parameter ranges concerning densities and sizes, and is a macromolecular generalization [36-38] of the interaction site description introduced by Chandler and Andersen for small rigid molecules [39]. This generalization has proven rather successful for pure especially dense polymer systems and polymer alloys. Some results for dilute particle mixture systems have been obtained within a simplification of the approach $[40,41]$ and could rationalize several surprising aspects of measured second virial coefficients of small proteins [20]. Also, light scattering measurements of the colloid liquid structure could be 
described semiquantitatively over all length scales without adjustable parameters [35].

In the present paper we analyze in detail the low-density limits of this macromolecular approach. The reasons are threefold. First, by looking at polymer solutions containing few colloidal particles, it is possible to compare with exact field-theoretic results and thus to test the approach. Second, by considering dilute polymers in a hard sphere solvent it is possible to make contact with the previous Asakura-Oosawatype approaches. Third, in these limits fully analytical solutions of the nonlinear integral equations description are possible and provide insight into the theory, which also applies to the higher concentration states.

A conceptually new closure (approximation) for the direct correlation function describing the packing of polymers close to repulsive walls or around hard colloidal spheres has been introduced in [35], which entails a medium-ranged colloidpolymer segment effective interaction. To capture the key physics the latter is required within the polymer reference interaction site model (PRISM) approach since a preaveraging approximation for the single-polymer-chain form factor is employed for tractability reasons. In inhomogeneous systems, however, the single-polymer form factor depends on the distance of the polymer chain from interfaces or inhomogeneities. Considering a fluid of random walk polymers, Gaussian intramolecular correlations apply. The number of intersections of a random walk with a plane scales as $\sqrt{N}$, where $N$ is the number of steps or polymer repeat units. Without rearrangements, the number of contacts with a repulsive wall would scale identically, as the excluded volume constraint could be satisfied by just mirror inverting the overlapping polymer strands. This result follows from PRISM with the most simple excluded volume closure (of the Percus-Yevick form) [40]. Close to the repulsive wall, however, the (Gaussian) intramolecular polymer correlations differ from the ones in the bulk solution as translational entropy can be gained by reducing the number of contacts with the wall to $O(1)$, as required for recovery of the ideal gas equation of state from the wall virial theorem. In order to describe the inhomogeneous system with one homogeneous polymer intramolecular structure factor, the rearrangements close to a colloidal particle need to be captured by an effective colloidpolymer interaction that extends across the range where the polymer segments rearrange. We proposed a molecular closure convoluting the local (bare) segmental steric repulsion with a Yukawa weight where the range, or nonlocality length, called $\lambda$ is determined from thermodynamic consistency considerations. On the segmental level the assumption of shortranged effective steric interactions entered into a PercusYevick style approximation [40,41].

Thermodynamic consistency correlates the structure on local length scales with long wavelength fluctuations and is a familiar concept in liquid state theories [42]. In the present case, the polymer chemical potential at infinite dilution, closely connected to the insertion free energy for adding dilute polymers to a particle fluid, is used to implement consistency as it provides one of the simplest measures of the tendency of colloids and polymers to mix. Moreover, this quantity also determines the free volume, which is one of the input quantities in the colloid theory most widely used for large colloid to polymer size ratios [5].

The outline of this paper is as follows. In Sec. II the model of colloid-polymer mixtures is presented. Section III describes the solution of the integral equations in the two low-density limits of interest. The thermodynamic consistency equations are solved and discussed in Sec. IV. Section $\mathrm{V}$ then presents the results and discussions for the structure and thermodynamics of dilute colloidal particles in a polymer solvent, while Sec. VI describes the opposite case of dilute polymer chains immersed in a hard sphere fluid. Conclusions are presented in Sec. VII, and three appendices contain technical material and a discussion of alternative closure approximations.

\section{MODEL}

The binary mixture shall be described by its (matrix of partial) structure factors $\hat{S}_{i j}(q)$, where the index $i=1$ indicates the polymer and 2 indicates the colloid component. A small molecule solvent is treated as a background continuum and enters only implicitly via the interaction potentials for the polymers and colloids. In principle, all partial structure factors are experimentally measurable by (labeling and) scattering techniques. The total density fluctuations are decomposed into single-molecule contributions, described by a (diagonal) intramolecular form factor $\hat{\omega}_{i j}(q)=\hat{\omega}_{i}(q) \delta_{i j}$ and intermolecular correlations $\hat{h}_{i j}(q)$ resulting in

$$
\hat{S}(q)=\varrho \hat{\omega}(q)+\varrho \hat{h}(q) \varrho .
$$

An obvious matrix notation is used. The diagonal matrix of densities $\varrho_{i j}=\varrho_{i} \delta_{i j}$ gives the number density of colloidal particles and polymer segments. The pair decomposable excluded volume or steric interaction prevents the particles/ segments from overlapping,

$$
g_{i j}\left(r<\frac{1}{2}\left(\sigma_{i}+\sigma_{j}\right)\right)=0,
$$

where $\sigma_{2}=\sigma_{c}$ is the colloidal hard-core diameter and $\sigma_{1}$ $=\sigma_{p}$ is the excluded volume diameter of a single-polymer repeat unit (segment). The intermolecular pair correlation functions $g_{i j}(r)$ are trivially connected to the total intermolecular correlation functions $h_{i j}, g_{i j}(r)=h_{i j}(r)+1$. Carets in Eq. (1) denote Fourier-transformed quantities. The total density fluctuations of the interacting fluid are decomposed into the single-molecule fluctuations and an interaction part via a generalized Ornstein-Zernicke, or Chandler-Andersen, equation $[38,39,43]$,

$$
\hat{S}^{-1}(q)=\hat{\omega}^{-1} \varrho^{-1}-\hat{c}(q) .
$$

In a preaveraging approximation the single-polymermolecule density fluctuations

$$
\hat{\omega}_{p} \equiv \omega(q)=\frac{1}{N} \sum_{\alpha \beta}^{N}\left\langle e^{i \mathbf{q} \cdot\left(\mathbf{r}_{\alpha}-\mathbf{r}_{\beta}\right)}\right\rangle
$$


are taken to be known a priori. As the colloidal particle is assumed to be rigid and solid, it acts as a point scatterer, $\hat{\omega}_{c}=1$, subject to the steric restriction, Eq. (2). If the effective interaction potentials, the direct correlation functions, $c_{i j}(r)$ in Eq. (3), were taken to be the bare pair potentials, $c_{i j}(r)=-V_{i j}(r) /\left(k_{B} T\right)$, then Eqs. (1) and (3) would correspond to the random-phase approximation (RPA), which is one of the simplest liquid state approximations for (dense) polymeric and simple fluids. Typically one finds that RPA solutions violate the excluded volume condition, Eq. (2). Integral equation approaches like PRISM go beyond the RPA as they enforce the no-overlap condition Eq. (2) rigorously and determine the direct correlation functions from selfconsistency equations implementing the (physically) motivated expectation that the $c_{i j}(r)$ are short ranged and vanish beyond a few particle diameters. For the colloidal hard sphere component this corresponds to the well-established Percus-Yevick (PY) approximation [42]

$$
c_{c c}\left(r>\sigma_{c}\right)=0 .
$$

This closure and the excluded volume constraint, Eq. (2), together with the site-site Ornstein-Zernicke equation, Eq. (3), result in a coupling of density fluctuations at different wave vectors, thus leading to nonlinear integral equations with a much richer mathematical structure than the simple RPA. For the model of hard spheres, the only thermodynamic parameter is the packing fraction $\phi_{c}=(\pi / 6) \varrho_{c} \sigma_{c}^{3}$.

Detailed studies of the PRISM equations for homopolymer solutions and melts [37,38] have established that the polymer site-site direct correlation function to a good approximation decays to zero beyond the polymer repeat unit size so that a correspondingly simple closure can be enforced,

$$
c_{p p}\left(r>\sigma_{p}\right)=0 .
$$

Thus, the interaction between polymer macromolecules is made up of pairwise site-site segmental interactions, which are given by a spherically symmetric effective potential, which follows from the excluded volume constraint, Eq. (2). Attractive interactions beyond the "athermal" model studied here can be included $[38,41]$. Site averaged quantities are considered and therefore specific chain-end effects are neglected.

As discussed in the Introduction, the effective colloidpolymer interaction extends beyond the range of immediate overlap. This arises because of, and allows to accommodate, the change of the polymer conformations close to colloidal particles. As the exact direct correlation function is not known we suggested [35] a simple one-parameter extension of the PY closure (called modified PY, $m$-PY),

$$
\hat{c}_{c p}(q)=\frac{\hat{c}_{c p}^{s}(q)}{1+q^{2} \lambda^{2}}
$$

with

$$
c_{c p}^{s}\left(r>\frac{\sigma_{c}+\sigma_{p}}{2}\right)=0,
$$

which enforces excluded volume on the local scale by fixing $c_{c p}^{s}(r)$ from the excluded overlap condition, Eq. (2), and from the requirement of short-ranged segmental interactions. On physical grounds, because $-k_{B} T c_{c p}^{s}(r)$ describes interactions on the segmental scale, one expects $c_{c p}^{s}(r)$ to be negative (repulsive) and to exhibit rapid variations (on the segmental length scale) and smoother ones connected with the colloid size. In real space the closure clearly implies a smearing of the segment-colloid interactions over the distance $\lambda$,

$$
c_{c p}(\mathbf{r})=\int d^{3} s \frac{1}{4 \pi \lambda^{2}} \frac{1}{|\mathbf{r}-\mathbf{s}|} e^{-|\mathbf{r}-\mathbf{s}| / \lambda} c_{c p}^{s}(\mathbf{s}),
$$

where the PY closure for $c_{c p}^{s}(r)$ can be viewed as describing unconnected polymer segments, and the (nonlocal) conformational constraints on the segment packing ("chain connectivity") close to colloidal particles are captured by the spatial convolution.

The $m$-PY closure contains an undetermined parameter, the length $\lambda$, which can be expected to vary nontrivially with the physical system parameters, like densities or size ratio. As it captures the rearrangements of the polymers strands close to a colloidal particle, its magnitude should be of the order of or smaller than the polymer correlation length (i.e., radius of gyration $R_{g}$ for dilute systems, blob diameter or mesh size for semidilute conditions) and/or the colloid size. Also, the polymer conformational changes, and hence $\lambda$, will depend on the volume taken up by the colloidal spheres. In order to achieve a parameter-free a priori description, thermodynamic consistency shall be enforced to determine $\lambda$ uniquely. The implementation of this well-known concept within liquid state theories starts from the observation that the PY closure [38], $\lambda=0$, leads to results for the solution free energies obtained from the compressibility theorem, $\left(\partial^{2} / \partial \varrho_{i} \partial \varrho_{j}\right) F^{\mathrm{ex}}=-k_{B} T \hat{c}_{i j}(0)$, where $F^{\mathrm{ex}}$ is the excess free energy per unit volume, which compare favorably with fieldtheoretic results where available [40]. Thus, the excess chemical potential for inserting polymers into a hard sphere fluid, where $\delta \mu_{i}=\left(\partial / \partial \varrho_{i}\right) F^{\mathrm{ex}}$, as obtained via the "compressibility" route provides a rather $\lambda$-insensitive reference quantity since it emphasizes long wavelenth correlations. Especially, the limit for vanishing polymer concentration shall be discussed,

$$
\left.N \beta \delta \mu_{p}^{(\mathrm{c})}\right|_{\varrho_{p}=0}=-\left.\int_{0}^{\varrho_{c}} d \varrho_{c}^{\prime} N \hat{c}_{c p}\left(q=0, \varrho_{c}^{\prime}\right)\right|_{\varrho_{p}=0},
$$

where $\beta=1 /\left(k_{B} T\right)$, and the expression per molecule is given. An independent, more local route to the insertion free energy will lead to strongly $\lambda$-dependent results, thereby allowing a sensitive determination of $\lambda$ from equating both expressions. The approach of thermodynamic integration introduced for RISM approaches by Chandler [43] shall be used as it connects the pair correlation functions on local distances to the 
thermodynamic properties. The variation of the free energy when turning on the interactions via the Mayer $f$ function, $f_{\alpha \beta}^{(\zeta)}: \zeta \in[0,1] \mapsto f_{\alpha \beta}^{(\zeta)}$, shall be used, where $f_{\alpha \beta}^{(1)}$ is the physical function and $f_{\alpha \beta}^{(0)}$ belongs to some known reference system; here the Greek indices run over the colloid and the polymer segment sites. As only excluded volume interactions are present, and because the limit of vanishing polymer segment size, $\sigma_{p} \rightarrow 0$, is of interest, the thermodynamic integration is chosen to take a mixture of polymers and colloidal point particles of free energy $F_{0}$ to the true mixture by growing the colloidal particles, $\sigma_{c}^{(\zeta)}=\zeta \sigma_{c}$. From Ref. [43] one then easily finds

$$
\begin{aligned}
\beta\left(F-F_{0}\right)= & \frac{\pi \varrho_{c} \varrho_{p} \sigma_{c}}{2} \int_{0}^{1} d \zeta\left(\sigma_{p}+\zeta \sigma_{c}\right)^{2} g_{c p}^{(\zeta)}\left(\frac{\sigma_{p}+\zeta \sigma_{c}}{2}\right) \\
& +2 \pi \varrho_{c}^{2} \sigma_{c}^{3} \int_{0}^{1} d \zeta \zeta^{2} g_{c c}^{(\zeta)}\left(\zeta \sigma_{c}\right)
\end{aligned}
$$

where the $g^{(\zeta)}$ are the $\zeta$-dependent (e.g., via the volume fraction of the colloid particles) pair correlation functions that are evaluated at the distances of closest approach. Equation (9) expresses that the growing colloidal spheres have to push against the pressure of the surrounding system (polymers and colloids), which - in a virial theorem analogy-is given by the probability of contact on the surface. Immediately, one obtains a second independent result for the chemical potential of Eq. (8), which, as argued, depends on $\lambda$ strongly as the packing of polymer segments close to the colloidal particles, $g_{c p}\left(\frac{1}{2}\left[\sigma_{c}+\sigma_{p}\right]\right)$, enters crucially,

$$
\begin{aligned}
\left.N \beta \delta \mu_{p}^{(\mathrm{g})}\right|_{\varrho_{p}=0}= & \frac{\pi \varrho_{c} \sigma_{c} N}{2} \int_{0}^{1} d \zeta\left(\sigma_{p}+\zeta \sigma_{c}\right)^{2} \\
& \times\left. g_{c p}^{(\zeta)}\left(\frac{\sigma_{p}+\zeta \sigma_{c}}{2}\right)\right|_{\varrho_{p}=0} \\
& +\left.2 \pi \varrho_{c}^{2} \sigma_{c}^{3} N \int_{0}^{1} d \zeta \zeta^{2} \frac{\partial g_{c c}^{(\zeta)}\left(\zeta \sigma_{c}\right)}{\partial \varrho_{p}}\right|_{\varrho_{p}=0} .
\end{aligned}
$$

Even though it would be desirable to obtain $\lambda$ for all concentration ranges, its form especially for low polymer concentrations is required. On the one hand, even small amounts of polymers added to colloidal systems can strongly affect the phase diagram and colloid structure. On the other hand, if $\lambda$ 's dependence on the polymer parameters is known then scaling considerations allow reasonable extrapolations of $\lambda$ into the semidilute region as will be shown in Sec. V. Therefore, the two expressions, Eqs. (8) and (10), for vanishing polymer concentrations will be used to obtain $\lambda$ in Sec. IV.

\section{SOLUTION IN LOW-DENSITY LIMITS}

The specified model of colloid-polymer mixtures covers all polymer and colloid density regions, and distances from the polymer repeat unit size (ca. $5 \AA$ ) up to the collective correlation length approaching phase separation (some micrometers). Since small amounts of nonadsorbing polymer can alter the structure and phase diagram of a colloidal system quite appreciably, simplification to consider rather low polymer concentrations is of initial interest. Under these conditions there opens up a mesoscopic window where polymer segments are well separated but polymer molecules overlap and interact strongly. Such solutions are called dilute or semidilute [44], and treatment of this regime is most conveniently done by performing the "thread limit" $[45,46]$, where the size of a polymer segment and the corresponding statistical segment size $l_{p} / \sqrt{12}$ are taken to be negligibly small; $\sigma_{p} \propto l_{p} \rightarrow 0$. In order to retain polymer molecules with a finite radius of gyration $R_{g}$, the number of repeat units is increased beyond bounds, $N \rightarrow \infty$, such that $\xi_{0}^{2}=l_{p}^{2} N=R_{g}^{2} / 2$ remains fixed. As has been shown by a rigorous solution of the PRISM integral equations in [47], intermolecular excluded volume remains active if in parallel the monomer density is increased, $\varrho_{p} \rightarrow \infty$, such that the number of polymer molecules per coil volume $\left(\sim R_{g}^{3}\right)$ stays finite: $\varphi_{p}$ $=2 \pi\left(\varrho_{p} / N\right) \xi_{0}^{3}$ is fixed. The reduced polymer concentration $\varphi_{p}$ differs only by a numerical factor from the often used polymer packing fraction $\eta_{p}=(4 \pi / 3)\left(\varrho_{p} / N\right) R_{g}^{3}=\varrho_{p} / \varrho_{p}^{*}$ $\approx \varphi_{p} / 0.53$, where $\varrho_{p}^{*}$ is the density when polymer coils start to interpenetrate. The mathematical thread limit of the PRISM equations corresponds to a scaling law description of the dilute-to-semidilute crossover of polymer solutions and can be compared to field-theoretic scaling laws and results [48]. In both cases only mesoscopic parameters, the polymer molecule density and coil size, enter and all microscopic parameters, like $\sigma_{p}, l_{p}$, and $\varrho_{p} \sigma_{p}^{3}$, drop out. The effective polymer-polymer interaction becomes of the Edwards $\delta$-function type, $c_{p p}(r)=\hat{c}_{p p}(0) \delta(\mathbf{r})$, where the intermolecular excluded volume parameter $\hat{c}_{p p}(0)$ follows selfconsistently from the no-overlap condition, Eq. (2). Besides its use for dilute and semidilute polymer solutions, experience also has shown, when applying the thread limit outside its rigorous range of validity, that it describes qualitatively adequately the spatially coarse-grained features of concentrated polymer solutions and melts [49].

Two limits of the scaling function of the single-chain form factor are known in general, and for Gaussian polymers simplify to $\omega(q=0)=N$ and $\omega\left(q R_{g} \gg 1\right) \rightarrow\left(q l_{p}\right)^{-2}$. Note that the self-scattering term, which is present in the full $\omega(q)$ of Eq. (4) for $q \sigma_{p}=O(1)$, is not accessible in the thread limit. In order to keep simple and analytically tractable equations, the full intramolecular structure factor shall be approximated by the standard Padé interpolation between the two asymptotes,

$$
\omega(q) \approx \frac{N}{1+q^{2} \xi_{0}^{2}} .
$$

As the single-polymer structure factor is an input to our PRISM approach, the use of random walk statistics in Eq. (11) for repelling coils can be considered an additional technical approximation in order to achieve analytical results. In order to capture effects of the nontrivial intramolecular cor- 
relations ("swelling" and "self-avoiding-walk statistics") caused by intramolecular excluded volume, Eqs. (1) to (10) could be solved numerically with an appropriate $\omega(q)$ [48]. Also, effects specific to semiflexible polymers and arising from local chain rigidity are neglected in Eq. (11), but could be incorporated into numerical studies.

In this section as well as in Sec. IV, in the appendices and in the figure captions, dimensionless units shall be chosen by using the colloid diameter as unit of length, $\sigma_{c}=1$. Then, the length scale ratio $\xi_{0}=R_{g} /\left(\sqrt{2} \sigma_{c}\right)$, the relative polymer concentration $\varphi_{p}$, and the colloid packing fraction $\phi_{c}$ are the only remaining physical parameters. Further notational simplification is provided by defining $\bar{S}_{c c}=S_{c c} / \varrho_{c}, \quad \bar{S}_{p p}$ $=\left(l_{p}^{2} / \sigma_{c}^{2} \varrho_{p}\right) S_{p p}, \bar{c}_{c p}^{s}=c_{c p}^{s} \sigma_{c}^{2} / l_{p}^{2}$, and $\bar{\omega}(q)=\left(l_{p} / \sigma_{c}\right)^{2} \omega(q)$.

The limit of considering only (semi-) dilute polymer solutions does not eliminate the nonlinearities of the integral equations for the polymer and colloid structure. Insights into the physics described by the $m$-PY PRISM equations and their full solutions can be gained by reducing one of the densities further to a dilute limit where at most pairwise direct interactions of the diluted species can occur. This linearizes the equations in the correlation functions of the diluted species and thus, as the correlations of the majority component are known, simplifies the analysis. These limits will be studied in the following, where in Sec. III, the $m$-PY closure parameter $\lambda$ still is kept arbitrary.

\section{A. Dilute colloids}

In the limit $\phi_{c} \rightarrow 0$ the equations simplify as the colloid particles do not alter the structure of the polymer fluid. The collective polymer structure factor for Gaussian intramolecular correlations within PRISM equals [38]

$$
\hat{\bar{S}}_{p p}=\frac{\xi^{2}}{1+q^{2} \xi^{2}}
$$

where the polymer correlation length crosses over from $\approx R_{g}$ at high dilution to the blob size or density screening length for concentrations within the semidilute regime,

$$
\frac{1}{\xi}=\frac{1}{\xi_{0}}+\frac{1}{\xi_{\varrho}}=\frac{1+2 \varphi_{p}}{\xi_{0}}
$$

Note that the neglect of microscopic length scales corresponds to the assumption that the polymer concentrations in Eq. (13) are far smaller than melt densities, where segments of different polymer chains start to pack densely (typically $\approx 30-40 \%$ of melt density).

The polymer segment profile close to a single colloidal particle and the resulting packing of two colloidal hard spheres is described by linear equations

$$
\hat{h}_{c p}(q)=\frac{\hat{\bar{c}}_{c p}^{s}(q)}{1+q^{2} \lambda^{2}} \hat{\bar{S}}_{p p}(q)
$$

and

$$
\hat{h}_{c c}(q)=\hat{c}_{c c}(q)+\frac{\varphi_{p}}{2 \pi \xi_{0}} \frac{\hat{\bar{c}}_{c p}^{s}(q)}{1+q^{2} \lambda^{2}} \hat{h}_{c p}(q)
$$

Closed equations for the pair correlation functions follow from Eqs. (2), (14), and (5)-(7). Even though these equations can be solved by straightforward conversion to differential equations, a simplified Wiener-Hopf factorization of Eq. (14) is used in Appendix A because it is close to the solution technique of the full equations for arbitrary $\phi_{c}$ and $\varphi_{p}$ (to be published elsewhere), and can be presented in a more concise way. In Appendix A 1 it is shown that the functions $f_{i j}(r)=r\left(g_{i j}(r)-1\right)$ satisfy simple differential equations. The one describing the polymer segment density profile close to a single sphere is

$$
\left(1+\lambda \partial_{r}\right)\left(1+\xi \partial_{r}\right) f_{c p}(r)=0 \quad \text { for } r \geqslant \frac{1}{2},
$$

with initial conditions $f_{c p}^{\prime}\left(\frac{1}{2}\right)=g_{c p}^{\prime}\left(\frac{1}{2}\right) / 2-1=-1$ and $f_{c p}^{\prime \prime}\left(\frac{1}{2}\right)=g_{c p}^{\prime \prime}\left(\frac{1}{2}\right) / 2=\left(\frac{1}{2}+\lambda+\xi\right) /(\lambda \xi)$, where a prime denotes a derivative. Also the direct correlation function at zero wave vector is obtained from Eqs. (A3) and (A9),

$$
\begin{aligned}
\hat{c}_{c p}(0)= & \frac{-\pi \xi_{0}^{2}}{6 N \xi^{2}}\left[1+6 \xi+12 \xi^{2}+6 \lambda(1+2 \xi)^{2}\right. \\
& \left.+12 \lambda^{2}(1+2 \xi)\right] .
\end{aligned}
$$

For dilute polymer solutions, $\varphi_{p} \rightarrow 0$, the quantity $-N \hat{c}_{c p}(0)$ is the cross second virial coefficient describing the mutual excluded volume between a hard sphere and a Gaussian polymer coil.

For the pair correlation function describing the probability of two isolated spheres in the polymer solution to be at a separation $r$, the differential equation is

$$
\left(1+\lambda \partial_{r}\right) f_{c c}(r)+\frac{\varphi_{p}}{\xi_{0}}\left(u+v \lambda \partial_{r}\right) f_{c p}\left(r-\frac{1}{2}\right)=0 \quad \text { for } r \geqslant 1,
$$

with initial condition $f_{c c}(1)+1=g_{c c}(1)$. The unknown parameters $u$ and $v$ follow from Eqs. (A9) and (A11), and the initial value is given by the colloid pair contact value at infinite dilution, $\phi_{c} \rightarrow 0$,

$$
g_{c c}(1)=1+\frac{\varphi_{p}\left(2 \xi^{2}-4 \lambda^{3}(1+2 \xi)+4 \lambda \xi(1+2 \xi)+\lambda^{2}\left[3+4 \xi-4 \xi^{2}\right]\right)}{4 \xi^{2} \xi_{0}},
$$

where corrections of the order $O\left(e^{-1 / \lambda}\right)$ were neglected. The contact probability for two colloids in principle can be calculated 
exactly within $m$-PY, but the approximation to neglect corrections of $O\left(e^{-1 / \lambda}\right)$ simplifies the expressions greatly, and for realistic values, (see below) where $\lambda<$ (mostly $\ll$ ) 0.31 , introduces errors of at most a few percent.

From Eq. (15) and the initial conditions, it follows that $g_{c p}(r)$ consists of a superposition of two Yukawa tails, $A_{\xi} e^{-r / \xi} / r$ and $A_{\lambda} e^{-r / \lambda} / r$, where $A_{\xi}>0$ and $A_{\lambda}<0$ for $\lambda$ $<\xi$, and that it describes a monotonous increase from zero to unity for $\frac{1}{2} \leqslant r<\infty$. Similarly, Eq. (17) shows that $g_{c c}(r)$ is a superposition of these two Yukawa tails (with constants $B_{\xi}$ and $B_{\lambda}$ ) and of an additional secular term $C_{\lambda} e^{-r / \lambda}$. Nevertheless, numerically it is found to decrease monotonously for all parameters.

Of interest is the second virial coefficient $B_{2}^{c}$, which follows from the (colloid partial) compressibility and measures the strength of the polymer induced pair potential [50],

$$
\begin{aligned}
B_{2}^{c} & =\left.\frac{-1}{2} \int d^{3} r h_{c c}(r)\right|_{\varrho_{c}=0} \\
& =B_{2}^{\mathrm{HS}}\left[1-\left.3 \int_{1}^{\infty} d r r f_{c c}(r)\right|_{\varrho_{c}=0}\right],
\end{aligned}
$$

where the result for hard spheres, $B_{2}^{\mathrm{HS}}=2 \pi / 3$, expresses a purely steric repulsion, and negative values of $B_{2}$ indicate the presence of a net attractive effective interaction due to "depletion." One finds $B_{2}^{c}$ easily from the solution of Eq. (17) with initial value given in Eq. (18) [neglecting corrections of order $\left.O\left(e^{-1 / \lambda}\right)\right]$ and parameters in Eqs. (A9) and (A11). For $\lambda=0$, the PY result of Ref. [40] is recovered. The virial coefficient, as it still contains the parameter $\lambda$, will be discussed in Sec. V after the determination of $\lambda$ from thermodynamic consistency.

\section{B. Dilute polymers}

In the limit of adding only a few polymers to a dense fluid of hard spheres, the $m$-PY PRISM equations simplify to

$$
\hat{h}_{c p}(q)=\frac{\bar{\omega}_{q} \hat{\bar{c}}_{c p}(q)}{1+q^{2} \lambda^{2}} \hat{\bar{S}}_{c c}(q)
$$

and

$$
\hat{h}_{p p}(q)=\omega_{q}^{2} \hat{c}_{p p}(q)+\frac{6 \phi_{c}}{\pi} \frac{\bar{\omega} \hat{\bar{c}}_{c p}(q)}{1+q^{2} \lambda^{2}} \hat{h}_{c p}(q),
$$

where the structure factor of the pure hard sphere fluid, $\hat{S}_{c c}$, is given in PY approximation. The unperturbed length scale characterizing the polymer correlations is given by $\xi_{0}$ as for an isolated polymer. Colloid mediated interactions arise as the polymer coil is forced to squeeze into the voids between the spheres. In Appendix A 2 it is shown that the functions $f_{i j}(r)=r\left(g_{i j}(r)-1\right)$ satisfy linear integrodifferential equations with known integration kernels. The average polymer segment density around colloidal spheres increases according to $\left(1+\lambda \partial_{r}\right)\left(1+\xi_{0} \partial_{r}\right) f_{c p}(r)=12 \phi_{c} \xi_{0}^{2} \int_{-1 / 2}^{1 / 2} d s q_{c p}(s) f_{c c}(r-s)$

for $r \geqslant \frac{1}{2}$, where the initial conditions are $f_{c p}^{\prime}\left(\frac{1}{2}\right)=g_{c p}^{\prime}\left(\frac{1}{2}\right) / 2$ $-1=-1$ and

$$
f_{c p}^{\prime \prime}\left(\frac{1}{2}\right)=g_{c p}^{\prime \prime}\left(\frac{1}{2}\right) / 2=\frac{1-\phi_{c}+2\left(\lambda+\xi_{0}\right)\left(1+2 \phi_{c}\right)}{2 \lambda \xi_{0}\left(1-\phi_{c}\right)^{2}} .
$$

Here, the hard sphere function $f_{c c}(r)$ is known [42] and the new factor function is given by

$$
q_{c p}(r)=\frac{a}{2}\left(r^{2}-\frac{1}{4}\right)+b\left(r-\frac{1}{2}\right) \quad \text { for }-\frac{1}{2} \leqslant r \leqslant \frac{1}{2}
$$

and vanishes outside this range; the parameters are given in Eq. (A22). Again, the direct correlation function at zero wave vector, which is needed for the thermodynamic calculation, can be obtained in explicit form (see Appendix A 2),

$$
\begin{aligned}
\frac{-6 N}{\pi} \hat{c}_{c p}(q=0)= & \frac{1}{1-\phi_{c}}+\frac{6\left(\xi_{0}+\lambda\right)}{\left(1-\phi_{c}\right)^{2}} \\
& +\frac{12\left(\lambda+\xi_{0}\right)^{2}\left(1+2 \phi_{c}\right)}{\left(1-\phi_{c}\right)^{3}} \\
& +\frac{24 \lambda \xi_{0}\left(\lambda+\xi_{0}\right)\left(1+2 \phi_{c}\right)^{2}}{\left(1-\phi_{c}\right)^{4}} .
\end{aligned}
$$

The polymer pair correlation function follows from Eqs. (A24) and (A27) for $r>0$

$$
\begin{aligned}
\left(1+\lambda \partial_{r}\right)\left(1+\xi \partial_{r}\right) f_{p p}(r)= & z_{\xi_{0}} e^{-r / \xi_{0}}+z_{\lambda} e^{-r / \lambda} \\
& +12 \phi_{c} \xi_{0}^{2} \int_{-1 / 2}^{1 / 2} d s \quad q_{c p}(s)(r-s) \\
& \times\left[g_{c p}(|r-s|)-1\right]
\end{aligned}
$$

where the initial conditions are $f_{p p}(0)=0$ and $f_{p p}^{\prime}(0)=-1$, and the parameters are given in Eq. (A26). Clearly, by means of the last term the colloidal spheres imprint their local packing structure onto the dilute polymers. In order to explicitly calculate the pair correlation functions, numerical integration of Eqs. (21) and (25) is most convenient.

Also of interest is the intermolecular excluded volume parameter that describes a colloid modified effective interaction for polymer segments on different chains,

$$
\frac{\hat{c}_{p p}(0) \xi_{0}}{-8 \pi l_{p}^{4}}=\frac{b \xi_{0}^{2}+z_{\lambda}+\bar{z}_{\xi_{0}}}{\lambda+\xi_{0}}
$$

where the parameters are given in Appendix A 2. It simplifies to $\hat{c}_{p p}=-8 \pi l_{p}^{4} / \xi_{0}$ for the pure polymer system and is connected to the polymer second virial coefficient via 
$B_{2}^{p}=-\frac{1}{2} \hat{h}_{p p}(q=0)=-\frac{1}{2} N^{2}\left(\hat{c}_{p p}(0)+\frac{6 \phi_{c}}{\pi}\left[\hat{c}_{c p}(0)\right]^{2} \hat{\bar{S}}_{c c}\right)$,

which becomes $B_{2}^{p}=-\frac{1}{2} N^{2} \hat{c}_{p p}(0) \propto R_{g}^{3}$ when no colloids are present.

\section{ENFORCEMENT OF THERMODYNAMIC CONSISTENCY}

The solutions for the structure of colloid-polymer mixtures open up two very different calculation routes to the thermodynamic properties. One, via the long wavelength fluctuations, termed "compressibility route," is suggested in Eq. (8) for the insertion free energy of polymers into hard sphere fluids. The second, Eq. (10), uses very local information captured in the contact values of the pair correlation functions. Within integral equation theories for the structure of many-body systems, both results generally do not coincide and in the $m$-PY closure this aspect of "thermodynamic consistency" is used to determine the effective interactions length scale $\lambda$ from equating both results.

The ideal gas result for adding point polymers, $\xi_{0} \rightarrow 0$, immediately follows from Eqs. (8) and (24) and the observation that $\lambda \propto \xi_{0}$ (corresponding to the polymer appearing as an inpenetrable small sphere to the colloid) has to hold in this limit: $\left.N \beta \delta \mu_{p}^{(\mathrm{c})}\right|_{\varrho_{p}=0}=-\ln \left(1-\phi_{c}\right)+O\left(\xi_{0}\right)$. This change in translational entropy when dissolving a point polymer in a sphere solvent of packing fraction $\phi_{c}$ is connected to the free volume fraction accessible to the polymer, $e^{-N \beta \delta \mu_{p}}=V_{f} / V$ $=1-\phi_{c}$. The free volume for finite colloid but vanishing polymer concentrations is a central quantity in the phase studies of the free volume approach by Lekkerkerker et al. [5]. In order to arrive at the same ideal gas limit from the polymer-colloid contact probability in Eq. (10), since $\left.\left(\partial / \partial \varphi_{p}\right) g_{c c}(1)\right|_{\varrho_{p}=0}=O\left(\xi_{0}^{-1}\right)$ as follows from Eq. (18), the following result has to hold for the contact probability of polymer segments with the colloidal surface:

$$
g_{c p}\left(\frac{1+\sigma_{p}}{2}\right)=\frac{1}{N} \frac{1}{1-\phi_{c}} \quad \text { for } \xi_{0} \rightarrow 0 .
$$

The contact value should be microscopically small, vanishing in the mesoscopic limit $N \rightarrow \infty$. In the thread $m$-PY PRISM limit described in Sec. II, where microscopic parameters were scaled away, the colloid-polymer pair correlation function agrees with this result. Thread PRISM correctly predicts on mesoscopic scales that $g_{c p}\left(\frac{1}{2}\right) \rightarrow 0$. In the case of (semi-) dilute polymer solutions a scaling connection of the segment-segment contact value to the mesoscopic scaling law description could be shown, which enabled one to estimate the microscopically small contact value from an evaluation of the mesoscopic pair correlation function at a microscopic separation [48]. From the parabolic polymer segment density profile close to a colloidal particle and Eq. (22), one realizes that within $m$-PY this again is possible. The mesoscopic pair correlation function (i.e., the thread $g_{c p}$ ) evalu- ated at a microscopic separation $\tilde{\sigma}_{p}$ because of $g_{c p}\left(\frac{1}{2}\right.$ $\left.+\tilde{\sigma}_{p} / 2\right)=\frac{1}{2} g_{c p}^{\prime \prime}\left(\frac{1}{2}\right) \tilde{\sigma}_{p}^{2}$, predicts the correct scaling behavior of the contact value, Eq. (28),

$$
g_{c p}\left(\frac{1+\sigma_{p}}{2}\right)=\frac{1}{2} \frac{\tilde{\sigma}_{p}^{2}}{\lambda \xi_{0}} \frac{1}{1-\phi_{c}} \quad \text { for } \xi_{0} \rightarrow 0,
$$

and thus recovers the ideal gas result for the chemical potential from the "wall virial" route. Evidently, however, the microscopic distance could only be calculated if the full PRISM equations also including microscopic length scales were solved. As this has not been achieved yet, in Ref. [35] for simplicity the distance $\tilde{\sigma}_{p}^{2}=2 l_{p}^{2}$ was chosen, because then the distance over which pointlike polymers rearrange close to a colloid sphere becomes numerically identical to the polymer correlation length, $\lambda=\xi_{0}$, for $\xi_{0} \rightarrow 0$. Note that this holds for all colloid packing fractions and that $\tilde{\sigma}_{p}$ has to be chosen only once.

It is the major difference of the $m$-PY closure to the previously studied PY description, where $\lambda=0$, and (as can be expected) to prior numerical solutions of the PRISM-PY equations [51,52], that thermodynamic consistency in $\delta \mu_{p}$ is possible in a scaling sense. For $\lambda=0$ in the PY case, the mesoscopic pair correlation function predicts a scaling of the colloid-polymer contact value as $g_{c p}\left(1+\sigma_{p} / 2\right)=\tilde{\sigma}_{p} /\left[\xi_{0}(1\right.$ $\left.\left.-\phi_{c}\right)\right]$ for $\xi_{0} \rightarrow 0$, thus violating Eq. (28) and the ideal gas insertion free energy. Obviously, the PY closure overestimates the segment density of polymers, even of an isolated polymer, close to walls or colloidal spheres, as a factor $\sqrt{N}$ too high contact probability is predicted. This comparison justifies the motivation and the physical interpretation of the $m$-PY closure in Sec. II. Additionally, the PY approximation $\lambda=0$ predicts a segment density profile, which increases much more strongly,

$$
g_{c p}^{\mathrm{PY}}(r)=\left[\frac{1}{\xi_{0}\left(1-\phi_{c}\right)}+\frac{2+4 \phi_{c}}{\left(1-\phi_{c}\right)^{2}}\right]\left(r-\frac{1}{2}\right)+O\left[\left(r-\frac{1}{2}\right)^{2}\right],
$$

compared to

$$
g_{c p}(r)=\frac{1}{2} g_{c p}^{\prime \prime}\left(\frac{1}{2}\right)\left(r-\frac{1}{2}\right)^{2}+O\left[\left(r-\frac{1}{2}\right)^{3}\right]
$$

from Eq. (22), and thus strongly underestimates the polymer induced depletion attraction. This follows since for small separation of two colloidal particles, PY predicts a linear increase of the polymer density in the gap, whereas the correct $[25,30]$ and $m$-PY result for Gaussian polymers is a quadratic increase.

Extension of the expansion for small $\xi_{0}$ leads to the higher order result $\lambda=\xi_{0}-4 \xi_{0}^{2}+\xi_{0}^{3} \tilde{\Lambda}\left(\phi_{c}\right)+\cdots$, where the dependence on the sphere fluid packing density indicates an anomalous behavior of $\lambda$ for $\phi_{c} \rightarrow 1$, i.e., one finds $\tilde{\Lambda}(0)$ $=16$ but $\tilde{\Lambda}\left(\phi_{c}\right) \rightarrow-144 /\left(1-\phi_{c}\right)^{2}$ for $\phi_{c} \rightarrow 1$. As $\lambda$ is restricted to positive values, a different expansion scheme is required for high colloid packing fractions and for large 
polymers or small colloidal particles. It is described in Appendix B. The calculation leads to $\lambda \rightarrow \Lambda\left(\phi_{c}\right)$ for $\xi_{0} \rightarrow \infty$, where

$$
\Lambda\left(\phi_{c}\right) \rightarrow \begin{cases}\frac{1}{\sqrt{5}+1}-0.80 \ldots\left(\phi_{c}+\cdots\right), & \phi_{c} \rightarrow 0 \\ \frac{1 / 3}{\sqrt{5}+1}\left(1-\phi_{c}\right)+0.78 \ldots\left[\left(1-\phi_{c}\right)^{2}+\ldots\right], & \phi_{c} \rightarrow 1,\end{cases}
$$

which verifies the interpretation of $\lambda$ as the range of polymer segment rearrangement, because it is always less than the smaller of the two molecular sizes, and becomes vanishingly small if no free volume is available for the polymers. Exact determination of $\lambda$ from Eqs. (8) and (10) for all parameter values is difficult because thermodynamic integrations are required. Thus we proposed the approximation to interpolate between the known exact limits [35],

$$
\lambda^{-1}=\xi^{-1}+\frac{1+2 \phi_{c}}{1-\phi_{c}} \frac{\lambda_{1}}{\sigma_{c}},
$$

where $\lambda_{1}=(\sqrt{5}+1), \xi=\xi_{0}$ for vanishing polymer concentration, and for convenience dimensional units are restored. This Padé approximation satisfies the thermodynamic consistency condition from Eqs. (8) and (10) up to relative errors of $15 \%$ for all parameter values, see Fig. 1. For small polymers, $\lambda$ follows the polymer correlation length. For large single-polymer coils, $\lambda$ is expected to be determined by the pure hard sphere fluid correlation length beyond which density fluctuations are screened, which is $\phi_{c}$ dependent. The result in Eq. (31) can be shown to be in excellent agreement with this intuitive idea.

The expression in Eq. (31) immediately suggests an extrapolation for $\lambda$ from its present calculation at vanishing polymer concentration to finite $\varphi_{p}$. If the polymer correlation length of the dilute situation, $\xi_{0}$, is replaced with the full

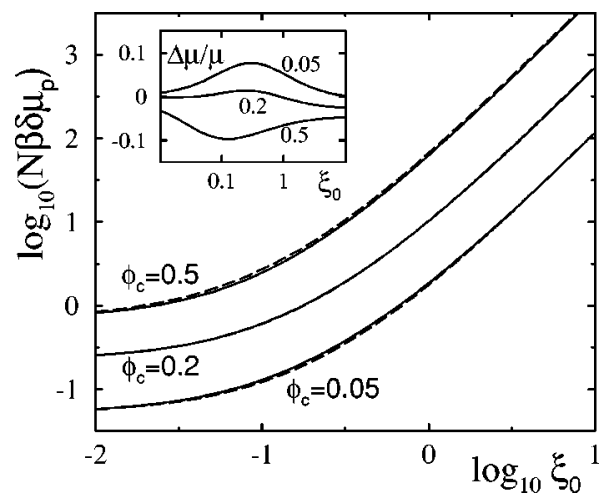

FIG. 1. Polymer molecule excess chemical potentials, $\left.\delta \mu_{p}\right|_{\varrho_{p}=0}$, as functions of the size ratio $\xi_{0}$ from the two different routes used to enforce thermodynamic consistency for three colloid packing fractions as labeled. The solid lines give the results from long wavelengths, Eq. (8), the dashed lines the corresponding ones from local packing, Eq. (10). The inset shows the relative errors, which for all parameters are smaller than $15 \%$. density dependent one, $\xi$, from Eq. (13), then $\lambda$ also is known for $\phi_{c}=0$ but now arbitrary polymer concentrations, $\varphi_{p}>0$. This replacement is suggested as the role of the single-polymer-molecule structure factor, $\omega(q)$, in Eq. (20) is taken over by the collective one, $S_{p p}(q)$, if the densities are changed accordingly. This standard procedure from polymer-scaling approaches thus substitutes the blob size or density screening length in place of the chain size for semidilute situations [44]. In Sec. VI, it will be shown that this extrapolation achieves thermodynamic consistency for the insertion free energy of adding colloids to a polymer solution, i.e., for quite a different thermodynamic quantity as originally considered for the determination of $\lambda$. In Ref. [35], this way of extrapolating $\lambda$ to finite polymer and colloid concentrations was suggested and used with $\xi$ $=\xi\left(\phi_{c}, \varphi_{p}, \xi_{0}\right)$ being the full polymer correlation length, which depends on $\phi_{c}, \varphi_{p}$, and $\xi_{0}$. In the two cases considered here it simplifies to $\xi\left(\phi_{c}, \varphi_{p}=0, \xi_{0}\right)=\xi_{0}$ and $\xi\left(\phi_{c}\right.$ $\left.=0, \varphi_{p}, \xi_{0}\right)=\xi_{0} /\left(1+2 \varphi_{p}\right)$, see Eq. (13).

\section{RESULTS FOR COLLOIDS DILUTED IN A POLYMER SOLVENT}

If only a small amount of colloidal hard spheres is dissolved in a polymer solution, then the structure of the polymer fluid is not affected. The intramolecular density fluctuations or the form factors for the individual polymers, $\omega(q)$, are rather well understood from field theoretic considerations [53]. PRISM describes the packing, i.e., the intermolecular correlations of the polymeric macromolecules that are simplified to Gaussian chain molecules in the present work. The intermolecular pair correlation function exhibits the wellknown "correlation hole," which shows that polymer molecules for entropic reasons softly repel each other. The correlation hole has a nontrivial structure on the length scale of the size of the molecule (radius of gyration) and also for shorter distances, on the mesh or blob scale characterized by the density screening length $\xi$.

Close to the diluted colloidal particle the polymer segment density is less than in bulk resulting in a depletion layer of varying width. Its form is described by Eq. (15) and shown in Fig. 2. The width is given by the polymer correlation length $\xi$ as long as the particle is much larger. Only for dilute polymer solutions, this correlation length agrees with the polymer size $\xi_{0}$. In the semidilute region, it is given by the blob size or mesh width. If the particle becomes smaller than the polymer correlation length, then the depletion layer 




FIG. 2. Polymer-colloid pair correlation function, $g_{c p}(r)$, exhibiting the polymer segment depletion layer close to an isolated colloidal sphere for various polymer correlation lengths $\xi$ as labeled. The inset shows a double logarithmic plot of the width $w$ of the depletion layer, defined by $g_{c p}\left(r=\frac{1}{2}+w\right)=\frac{1}{2}$, as a function of $\xi$; thin dashed lines mark the asymptotes $w \rightarrow 1.66 \xi(w \rightarrow 1.1)$ for $\xi$ $\rightarrow 0(\xi \rightarrow \infty)$, respectively.

width crosses over to the particle diameter. This is shown in the inset in Fig. 2. If very small particles are immersed in the polymer solution, then the depletion layer has an additional power law tail due to chain connectivity correlations, which becomes $g_{c p}(r \gg 1)=1-\left(\frac{1}{2}+1 / \lambda_{1}\right)\left(\sigma_{c} / r\right)$ for $\xi \rightarrow \infty$ [25].

In the dilute polymer solution limit, the PRISM result compares rather well with field-theoretic calculations from Ref. [25]. While the width is of the same order in both results, especially the small distance power-law increase of the density profile, $g_{c p}(r)=\frac{1}{2} g_{c p}^{\prime \prime}\left(\frac{1}{2}\right)\left(r-\sigma_{c} / 2\right)^{2}+\cdots$, can be compared as it obeys a scaling law, where the (universal) amplitude of PRISM (semi-) quantitatively agrees with the known field-theoretic (FT) limits,

$$
g_{c p}^{\prime \prime}\left(\frac{1}{2}\right) \rightarrow\left\{\begin{array}{lllll}
\frac{1}{\xi_{0}^{2}} & (\mathrm{PRISM}) & \frac{1}{\xi_{0}^{2}} & (\mathrm{FT}), & \xi_{0} \rightarrow 0 \\
\frac{2 \lambda_{1}}{\sigma_{c}^{2}} & (\mathrm{PRISM}) & \frac{8}{\sigma_{c}^{2}} & (\mathrm{FT}), & \xi_{0} \rightarrow \infty .
\end{array}\right.
$$

The PRISM result for the polymer profile in Eq. (15) extends into the semidilute concentration region where mean-field calculations for the case of $R_{g} \ll \sigma_{c}$ are available [30]. Again, the amplitude, $g_{c p}^{\prime \prime}\left(\frac{1}{2}\right) \rightarrow 1 / \xi^{2}$ for $\xi \rightarrow 0$, agrees verifying its universality $[25,26]$. Only in the case of very large polymers, $m$-PY overestimates the depletion effect by ca. $20 \%$ in Eq. (32). Figure 2 shows that the width of the depletion layer for semidilute solutions is set by the polymer correlation length (for $\xi \ll \sigma_{c}$ ), and that the molecular size $R_{g}$ plays no role for the depletion layer; this also is evident from Eq. (15) where only the correlation length of the collective polymer fluctuations appear. Using an effective pair potential approach $[1,4,5]$ determined under dilute polymer conditions strongly overestimates the range of the depletion interactions in semidilute polymer solutions.

The polymer induced depletion attraction becomes apparent from the colloid pair correlation function describing the probability of two isolated colloidal spheres to be at a dis-



FIG. 3. Colloid pair correlation function, $g_{c c}(r)$, for two isolated hard spheres in a solution of polymers with size ratio $\xi_{0}=5$ for the denoted polymer concentration $\varphi_{p}$. The inset shows as a solid line the range $w$ of the depletion attraction estimated by $g_{c c}(r=1+w)=\frac{1}{2}\left[1+g_{c c}(1)\right]$ as a function of the colloid-polymer interaction length $\lambda$. Curves for various polymer concentrations $\left(\varphi_{p}=0.1,1,2,10\right)$ overlap, while the size ratio $\xi_{0}$ runs between $0.01 \leqslant \xi_{0} \leqslant 100$. The dashed line compares the width of the depletion layer in $g_{c p}(r)$ from Fig. 2.

tance $r$, Eqs. (17) and (18). As seen in Fig. 3, for close distances and especially at contact, this probability is increased above the random value of unity. The $g_{c c}(r)$ decreases from the contact values, Eq. (18), monotonically without oscillatory features or layering. Thus, the effective or induced potential, $\beta V^{\text {eff }}(r)=-\ln g_{c c}(r)$, is attractive and monotonic, and does not exhibit repulsive barriers. The contact value scales like $g_{c c}(1)-1 \sim \varphi_{p} / \xi_{0}$ in either limit of very small or very large polymers, i.e., $\xi_{0} \rightarrow 0(\infty)$. The distance characterizing the decay of $g_{c c}(r)$ is closely connected to the width of the depletion layer as seen in the inset of Fig. 3 , and both depend on the polymer parameters only via the nonlocality length $\lambda$. The correlation between two colloidal particles that are much smaller than the polymer mesh width falls off for large distances as $g_{c c}(r \gg 1) \rightarrow 1+(1$ $\left.+2 / \lambda_{1}\right)^{2}\left(\pi l_{p}^{2} \sigma_{c}^{2} \varrho_{p} / r\right)$ for $\xi \rightarrow \infty$, indicating a weak but longranged attraction [27]. It becomes screened at the polymer or blob size only.

A measure of the tendency of colloidal spheres to dissolve in polymeric fluids is given by the chemical potentials or solvation energies. From the compressibility route in analogy to Eq. (8), one finds

$$
\left.\beta \delta \mu_{c}^{(\mathrm{c})}\right|_{\varrho_{c}=0}=-\left.\int_{0}^{\varrho_{p}} d \varrho_{p}^{\prime} \hat{c}_{c p}\left(q=0, \varrho_{p}^{\prime}\right)\right|_{\varrho_{c}=0}
$$

From the free energy [see Eq. (9)] required to grow the colloid particle from a point to its actual size one finds

$$
\left.\beta \delta \mu_{c}^{(\mathrm{g})}\right|_{\varrho_{c}=0}=\left.\frac{\pi c_{p} \sigma_{c}^{3} \xi_{0}^{2}}{2} \int_{0}^{1} d \zeta \zeta^{2} g_{c p}^{(\zeta) \prime \prime}(\zeta / 2)\right|_{\varrho_{c}=0}
$$

For dilute polymer solutions, where $\xi=\xi_{0}$, the result from both routes obeys thermodynamic consistency (up to errors of $10 \%$ ) and agrees with field-theoretic results up to $20 \%$ as shown in Fig. 4. From the local packing information, Eq. (34), an explicit result can be found, 


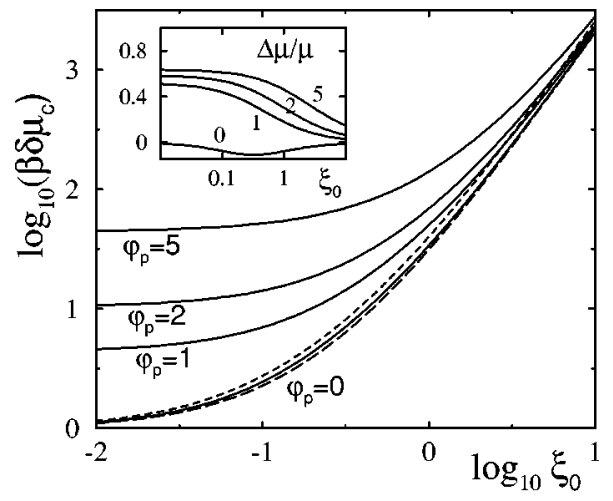

FIG. 4. Colloid excess chemical potentials at infinite dilution, $\left.\beta \delta \mu_{c}\right|_{\varrho_{c}=0}$, in units of $\left(\pi c_{p} / 6\right)$ as functions of the polymer to colloid size ratio $\xi_{0}$ for four polymer densities as labeled. The solid lines give the results from long wavelengths, Eq. (33), the long dashed line is the result from local packing, Eqs. (34) and (35), in the dilute polymer limit. The short dashed line compares the fieldtheoretic result known in the dilute limit [25]. The inset shows the relative errors in thermodynamic consistency for the four polymer densities.

$\left.\beta \delta \mu_{c}^{(\mathrm{g})}\right|_{\varrho_{c}=0}=\frac{\pi c_{p} \sigma_{c}^{3} \xi_{0}^{2}}{6 \xi^{2}}\left[1+\left(6+\frac{3}{2} \lambda_{1}\right)\left(\frac{\xi}{\sigma_{c}}\right)+6 \lambda_{1}\left(\frac{\xi}{\sigma_{c}}\right)^{2}\right]$,

which has a number of polymer specific features. For a fluid of small polymers or far into the semidilute regime, $\xi \rightarrow 0$, inserting a colloidal sphere costs the free energy of creating its volume by doing work against the osmotic pressure of the polymer fluid. For larger polymers, the form of the polymeric coil enters, and in the limit of very large polymer chains the chemical potential becomes independent of $R_{g}$ and scales linearly with the length over which polymer segments need to be rearranged, which is just the colloid diameter $\sigma_{c}$. As the added sphere sees local strands of the polymer network only, the result for small spherical colloids becomes independent of the polymer's size $\left(R_{g}\right)$ or degree of polymerization, and also independent of the mesh or blob size. Thus it becomes independent of the polymer molecule concentration [29],

$$
\left.\beta \delta \mu_{c}\right|_{\varrho_{c}=0} \rightarrow \pi \varrho_{p} \sigma_{c} l_{p}^{2} \lambda_{1} \quad \text { for } \xi \rightarrow \infty,
$$

which holds in the dilute and semidilute region. This result also quantitatively compares favorably to the known behavior in the dilute limit [25], $\left.\beta \delta \mu_{c}\right|_{\varrho_{c}=0} \rightarrow 4 \pi \varrho_{p} \sigma_{c} l_{p}^{2}$. For the semidilute region, the two routes in Eqs. (33) and (34) pre$\left.\left.\operatorname{dict} \beta \delta \mu_{c}^{(\mathrm{c})}\right|_{\varrho_{c}=0} \rightarrow \frac{1}{3} \beta \delta \mu_{c}^{(\mathrm{g})}\right|_{\varrho_{c}=0}$ and

$$
\left.\beta \delta \mu_{c}^{(\mathrm{c})}\right|_{\varrho_{c}=0} \rightarrow \frac{\pi}{6} \sigma_{c}^{3} \beta \Pi, \quad \beta \Pi=\frac{4}{3} c_{p} \varphi_{p}^{2} \text { for } \varphi_{p} \rightarrow \infty,
$$

which explicitly states the connection to the osmotic pressure $\Pi$ of the pure polymer system, which is given in the PRISM approximation for Gaussian polymers in Eq. (37). Note, that the extrapolation of $\lambda$ to finite polymer concentration leads

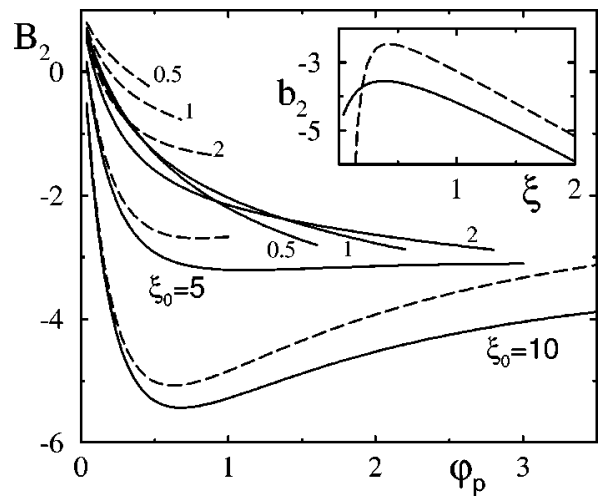

FIG. 5. Colloid second virial coefficient $B_{2}^{c}$ in units of the hard sphere result, $B_{2}^{\mathrm{HS}}=2 \pi / 3$, as a function of the polymer concentration for various size ratios $\xi_{0}$ as labeled. The solid lines present the results from long wavelengths, Eqs. (19), (38) and (39), the dashed lines present the results from local packing, Eq. (41). The curves are cut in order to prevent overcrowding the figure. The inset shows the semidilute scaling law, $\widetilde{b}_{2}^{c}(\xi)$ from Eq. (40), which applies to situations where the polymer correlation length $\xi$ (blob or mesh size) is of the order of the colloid diameter. The solid line again follows from Eq. (19) and the dashed one from Eq. (41).

to thermodynamic consistency also in these cases (as claimed at the end of Sec. IV), as both routes, Eqs. (33) and (34), lead to Eq. (36) identically and the results in Eq. (37) only differ by a numerical prefactor, which could be absorbed into a density dependent microscopic length $\tilde{\sigma}_{p}\left(\varphi_{p}\right)$.

The mean polymer induced depletion attraction for two colloidal spheres can be quantified using the second virial coefficient $B_{2}^{c}$, which is accessible experimentally. Representative results are shown in Fig 5 and demonstrate that the effective colloidal pair potential depends on the polymer concentration and the polymer to colloid size ratio. For small polymers, the result, obtainable from Eq. (19), simplifies to

$\frac{B_{2}^{c}}{B_{2}^{\mathrm{HS}}} \rightarrow 1-\frac{12 \varphi_{p}}{1+2 \varphi_{p}}+\frac{3\left(28 \lambda_{1}-53\right) \varphi_{p} \xi_{0}}{4\left(1+2 \varphi_{p}\right)^{2} \sigma_{c}}+\cdots \quad$ for $\xi_{0} \rightarrow 0$,

which indicates an appreciable attraction between the two colloids. Note that the third term is positive and predicts a weakening of the attraction when the polymer size starts to grow. Increasing the polymer density strengthens the attraction, which saturates at a finite negative value. The reliability of these results is discussed at the end of this section and in Appendix $\mathrm{C}$ where alternative closures are examined.

For very large polymers, again an effective two-colloid attraction is observed.

$$
\begin{aligned}
\frac{B_{2}^{c}}{B_{2}^{\mathrm{HS}}} \rightarrow & 1-\frac{3\left(2+\lambda_{1}\right)^{2}}{2 \lambda_{1}^{2}} \frac{\varphi_{p}\left(\xi_{0} / \sigma_{c}\right)}{\left(1+2 \varphi_{p}\right)^{2}} \\
& -\frac{\left(24+18 \lambda_{1}+3 \lambda_{1}^{2}\right) \varphi_{p}}{2 \lambda_{1}^{2}\left(1+2 \varphi_{p}\right)} \text { for } \xi_{0} \rightarrow \infty .
\end{aligned}
$$

As seen in Fig. 5, two hard spheres immersed in a fluid of much larger polymers feel an induced attraction, which var- 
ies nonmonotonically with polymer concentration and increases with polymer size as previously found based on the $\lambda=0$ PY closure [40]. Its origin is purely entropic as the added particles hinder conformational fluctuations of the polymer strand they are embedded in. For large distances, the conformational entropy loss at both particles is additive. For shorter distances, however, chain connectivity restricts the conformational fluctuations of a polymer molecule even without added particles. Thus the additional entropy loss is smaller if the two particles are close. This induces an effective long-ranged attraction among the colloidal particles. For small concentrations, adding polymer strengthens the effective attraction. Around the dilute-semidilute crossover density, however, the effective range of the polymer induced attraction crosses over from the polymer size to the mesh size and thus starts to decrease appreciably. The latter effect dominates in the semidilute concentration region and therefore $B_{2}^{c}$ decreases again. It is important to mention that a mean-field approach [53] employing a RPA, where $\xi$ $\sim 1 / \sqrt{\varrho_{p}}$, would be too crude to handle this competition and would miss the increase of $B_{2}^{c}$ above the overlap concentration. Apparently, a simple superposition approximation, which decouples the depletion layers around each of the colloid spheres, misses the long-ranged, but weak, attraction [54]. The minimum value of $B_{2}^{c}$ for the considered Gaussian polymer statistics is $-\left[3\left(2+\lambda_{1}\right)^{2} / 16 \lambda_{1}^{2}\right]\left(\xi_{0} / \sigma_{c}\right) \approx$ $-0.17\left(R_{g} / R_{c}\right)$, which is asymptotically deeper than the value $-0.50\left(R_{g} / R_{c}\right)^{0.40}$ for self-avoiding-walk polymer statistics [29]. The more swollen polymer molecules in good solvents apparently are more open to the particles and allow stronger interpenetration so that the induced colloid attraction is smaller [27,55].

Deep in the semidilute density region, $\varphi_{p} \rightarrow \infty$, the second virial coefficient again saturates, $B_{2}^{c} / B_{2}^{\mathrm{HS}} \rightarrow-5$, indicating a finite effect of the pairwise attraction, which is independent of the size ratio. This limit corresponds to a vanishingly small polymer blob size. Yet, for large polymers there can open up a window, where adding polymer only slightly changes $B_{2}^{c}$ in a manner that is much weaker than at the overlap concentration but still differs from the asymptotic value. This is the polymer concentration range where the polymer mesh width is not yet negligible compared to the particle size and there a broad maximum develops,

$$
\frac{B_{2}^{c}}{B_{2}^{\mathrm{HS}}} \rightarrow \widetilde{b}_{2}^{c}(\xi) \quad \text { for } \varphi_{p} \rightarrow \infty, \quad \xi_{0} \rightarrow \infty \quad \text { with } \xi=\text { const. }
$$

The scaling function $\tilde{b}_{2}^{c}$ is shown in the inset of Fig. 5. For semidilute polymer concentrations, the depletion attraction among two colloidal spheres depends nonmonotonically on the ratio of the blob to the sphere size. There is an optimal blob size that roughly equals the sphere radius, where the induced attraction is minimal or $B_{2}^{c}$ is maximal. This makes physical sense since for $\sigma_{c} / 2 \approx \xi$ the particles can "just fit in" the polymer mesh spaces without distorting it. For larger polymer correlation lengths, the crossover to Eq. (39) sets in because the range of attraction increases. Unexpectedly, however, also for smaller $\xi, B_{2}^{c}$ becomes more negative, presumably because the depth of the attraction increases.

There does exist a caveat for the above results on the colloid-colloid interactions $[7,9,35,40]$. Liquid state theory (and also [4]) with PY-closure, density functional approaches to the colloid structure, and free energy based approaches [5], all underestimate the depletion attraction in situations where it far exceeds $k_{B} T$. This error, which presumably affects the results for dilute colloidal particles in a dense solvent of small polymers, $\phi_{c} \rightarrow 0, \xi_{0} \ll \sigma_{c}$, and $\varphi_{p} \gg 1$, arises from an inherent linearization of the depletion potential in the considered binary-mixture approach. Therefore it is fortunate that a thermodynamic consistency condition can be formulated explicitly addressing the accuracy with which the depletion attraction is handled. There exists another independent expression for $B_{2}^{c}$, which follows from Eqs. (9) and (14) and the definition (19),

$$
\begin{aligned}
\frac{B_{2}^{c}}{B_{2}^{\mathrm{HS}}}= & 3\left[\int_{0}^{1} d \zeta \zeta^{2} g_{c c}^{(\zeta)}(\zeta)+\frac{\varphi_{p}}{48 \xi_{0}} \int_{0}^{1} d \zeta \zeta^{5} \frac{\partial g_{c p}^{(\zeta) \prime \prime}(\zeta / 2)}{\partial \phi_{c}}\right. \\
& \left.-\frac{\varphi_{p} \xi^{2}}{32 \xi_{0}}\left(\int_{0}^{1} d \zeta \zeta^{2} g_{c p}^{(\zeta) \prime \prime}(\zeta / 2)\right)^{2}\right]_{\varrho_{c}=0}
\end{aligned}
$$

This result follows from the local packing information and shows a very different and unphysical scaling compared with the result from large wavelength fluctuations for small polymer and/or high polymer concentrations, $B_{2}^{c(\mathrm{~g})} / B_{2}^{\mathrm{HS}}$ $\rightarrow \frac{1}{24}\left(\varphi_{p} / \xi_{0}\right)^{3}$. This throws severe doubts on the result in Eq. (38) and on our treatment of the depletion attraction in these two cases, $\xi_{0} \rightarrow 0$ or $\varphi_{p} \rightarrow \infty$, where from a pair potential point of view the depletion attraction should be arbitrarily large. Thus $m$-PY PRISM with the PY closure for colloidcolloid correlations apparently cannot capture the strong induced attraction in these two limits of theoretical interest as has been pointed out previously $[35,40]$. As the second virial coefficient can be considered a worst case example of this failure, Appendix $\mathrm{C}$ examines this issue using different theoretical approaches. Reassuringly, the results for the colloidal second virial coefficient for large polymers are recovered semiquantitatively from the two routes within $m$-PY PRISM. The scaling in the limit of very large polymers is recovered exactly, i.e., the term diverging linearly with $\xi_{0}$ in Eq. (39). Equation (41) also predicts that a scaling law exists for the second virial coefficient of colloidal particles in a polymer mesh, which semiquantitatively compares with the one of Eq. (40), see Fig. 5. Intriguingly it also shows the nonmontonic behavior of $\widetilde{B}_{2}^{c}(\xi)$ for intermediate $\xi$.

\section{RESULTS FOR POLYMERS DILUTED IN A HARD SPHERE FLUID}

While numerous field-theoretic results for polymer solutions exist, including for the question of dissolving one or two particles in dilute solutions, and could serve for tests of the $m$-PY results in the previous section, to our knowledge little is known about the packing of dilute polymer chains into dense particle fluids. The case of present interest is when 


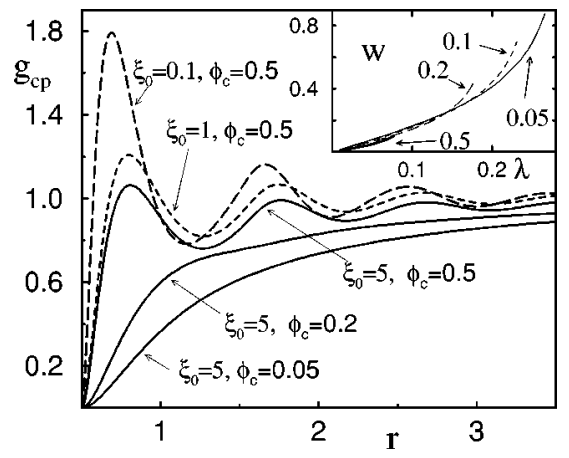

FIG. 6. Polymer-colloid pair correlation function $g_{c p}(r)$ for a polymer added to a hard sphere solution; colloid packing fraction $\phi_{c}$ and size ratio $\xi_{0}$ as labeled. The inset shows the width $w$ of the depletion layer [defined as $g_{c p}\left(r=\frac{1}{2}+w\right)=\frac{1}{2}$ ] versus the interaction range $\lambda$ for the labeled colloid packing fractions $\phi_{c}$. The curves are parametrized by the size ratio $\xi_{0}$, which varies as $0.01 \leqslant \xi_{0} \leqslant 100$ with increasing $w$ and $\lambda$.

the particles are much larger than the repeat units of the polymer chain, and both components are immersed in a small molecule background solvent treated as a continuum. If the amount of added nonabsorbing polymer is small, then the structure of the colloidal fluid is not changed. In the present treatment it is given by the reliable and easily improved PY-theory description.

Whereas the segmental depletion layer of a single polymer (or a semidilute solution) around a single (spherical) particle exhibits a monotonic dependence on the distance to the surface of the particle, adding further particles forces the polymer to squeeze into the open spaces. Thus the probability of finding polymer segments at a distance $r$ from the center of a colloidal particle, $g_{c p}(r)$, develops an oscillatory structure whose period is correlated with the colloidal size. In Fig. 6 the evolving layering of the segment density is shown. For high particle densities, the polymers pack tightly into the voids and thus are close to the particles. The depletion layer, even though present, in principle, is restricted by the colloid spacing and varies with external parameters. Thus, the assumption of an effective pair potential becomes

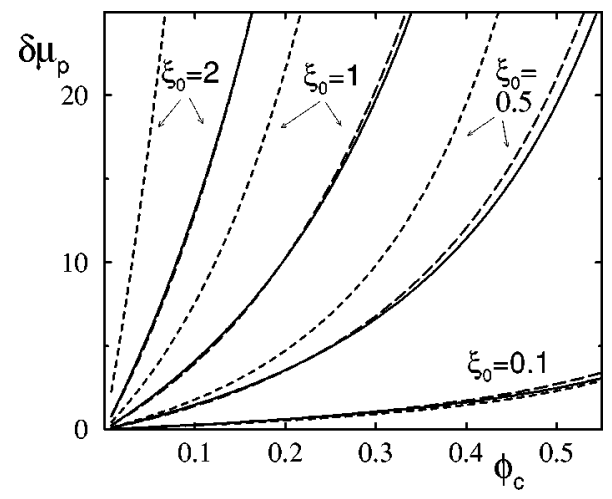

FIG. 7. Polymer excess chemical potentials at infinite dilution, $\left.\delta \mu_{p}\right|_{\varrho_{p}=0}$ [in units of $1 /(N \beta)$ ], as functions of the colloid packing fraction for four size ratios $\xi_{0}$ as labeled. The solid lines give the results from long wavelengths, Eq. (8), the long dashed lines the corresponding ones from local packing, Eq. (10). The short dashed lines compare the equivalent PY results for inserting a sphere of radius equal to $R_{g}$ as used in the phantom sphere approach of Ref. [5].

inappropriate, because it requires that the range of the polymer induced colloid-colloid potential, which naturally is connected to the depletion layer, itself becomes particle density dependent. The inset of Fig. 6 shows that there is a strong correlation of the colloid-polymer interaction range $\lambda$ to the width in the depletion layer. While varying the polymer size by four orders of magnitude, neither the width nor $\lambda$ change as strongly. Moreover, for somewhat higher colloid solvent concentrations the width becomes a unique function of $\lambda$, which only mildly splays out if $\phi_{c}$ is decreased. Both quantities furthermore arrest at finite values in the limit of $\xi_{0}$ $\rightarrow \infty$, since the relevant length scale is then the colloid size.

If the solvation free energy for adding nonadsorbing polymers to a fluid of spheres is considered, Eqs. (8) and (10), then the ideal gas limit result discussed in Sec. IV only holds for point polymers, $\xi_{0} \rightarrow 0$. As expected and shown in Fig. 7, it becomes more difficult to add larger polymers because less free volume is available for them. The compressibility expression, Eq. (8), can be integrated analytically yielding

$$
\left.N \beta \delta \mu_{p}^{(\mathrm{c})}\right|_{\varrho_{p}=0}=-\ln \left(1-\phi_{c}\right)+\frac{6 \phi_{c} \xi_{0}\left(1+\frac{4}{\lambda_{1}}\right)}{\sigma_{c}\left(1-\phi_{c}\right)}+\frac{6 \phi_{c} \xi_{0}^{2}\left(2+\phi_{c}\right)\left(1+\frac{2}{\lambda_{1}}\right)}{\sigma_{c}^{2}\left(1-\phi_{c}\right)^{2}}+\frac{6 \phi_{c} \xi_{0}\left(\frac{4}{\lambda_{1}^{2}}-\frac{2}{\lambda_{1}}\right) / \sigma_{c}}{\left(1+\lambda_{1} \frac{\xi_{0}}{\sigma_{c}}\right)\left[1+\lambda_{1} \frac{\xi_{0}}{\sigma_{c}}-\phi_{c}\left(1-2 \lambda_{1} \frac{\xi_{0}}{\sigma_{c}}\right)\right]},
$$

which was compared to the virial route in Fig. 1. For larger polymer coils, the added macromolecule loses conformational entropy when squeezing into the fluid interstitials and thus the free energy cost increases. Yet, its increase is not as rapid as if the polymer was a sphere because the polymer chain can rearrange. This becomes especially important for large polymers because they can wrap around the fluid particles. Therefore, if Eq. (42) is compared with the corresponding result from scaled particle or PY theory for the addition of spheres with size $R_{g}$ to a sphere fluid [5], then two qualitative differences appear for large $R_{g} \gg \sigma_{c}$. First, only a quadratic scaling with the polymer size results, 


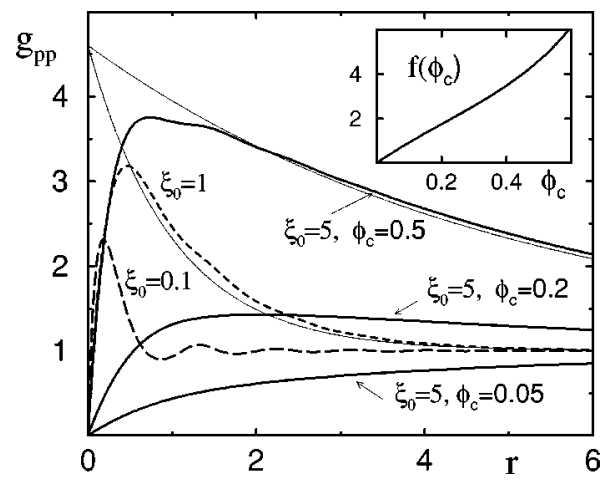

FIG. 8. Polymer-polymer pair correlation function $g_{p p}(r)$ for dilute polymer molecules immersed in a hard sphere solution; the colloid packing fractions $\phi_{c}$ and size ratios $\xi_{0}$ are the same as in Fig. 6 and as labeled ( $\phi_{c}=0.5$ for $\xi_{0}=0.1$ and 1). The thin lines present the asymptote for large polymers, Eq. (44), evaluated for $\xi_{0}=5$ and 1 at $\phi_{c}=0.5$, while the inset shows the intermolecular polymer segment contact value $f\left(\phi_{c}\right)$, which determines this asymptote.

$$
\left.\beta \delta \mu_{p}^{(\mathrm{c})}\right|_{\varrho_{p}=0} \rightarrow \frac{6 \phi_{c}\left(2+\phi_{c}\right)\left(1+\frac{2}{\lambda_{1}}\right)}{\left(1-\phi_{c}\right)^{2}} \frac{\xi_{0}^{2}}{N \sigma_{c}^{2}} \text { for } \xi_{0} \rightarrow \infty,
$$

whereas for (large) added spheres the chemical potential scales with the volume of the added species. The behavior in Eq. (43) is connected to the large- $\xi$ scaling of the chemical potential for adding spheres to a polymer solution, discussed in Eqs. (36) and (37), because both quantities are determined from the polymers' ability to deform around a particle. In Eq. (43) this explains why the right-hand side (rhs) becomes independent of the degree of polymerization and linearly dependent on the colloid size. Second, the increase with particle fluid density is weaker than the corresponding sphere mixture result, which would predict a cubic divergence for $\phi_{c} \rightarrow 1$. Thus the difference of both approaches becomes more important at higher sphere concentrations. Note that the free volume expression used in Ref. [5] is connected to $\left.\delta \mu_{p}\right|_{\varrho_{p}=0}$ via $\alpha=V_{f} / V=\left.e^{-N \beta \delta \mu_{p}}\right|_{\varrho_{p}=0}$. The input to this approach, the chemical potential for adding a sphere of radius $R_{g}$ to a hard sphere solution, is included in Fig. 7, and for large polymers strongly overestimates the free energy cost for insertion.

The pressure exerted by the particle fluid on the polymer also manifests itself in the dilute limit intermolecular packing of the segments of two polymers, which is described by $g_{p p}(r)$ for $\varphi_{p} \rightarrow 0$; examples are shown in Fig. 8. The potential-of-mean force between segments is given by $-k_{B} T \ln g_{p p}(r)$, while its polymer molecule analog is of the order of $N^{2}$ times larger. The slight repulsion of polymers in solution that causes the correlation hole is overcome at the distance connected to the depletion layer in $g_{c p}$, as seen by comparison with Fig. 6. For larger distances the polymer segments are pushed together and therefore pack more densely than random. For small polymers an oscillatory pattern develops connected to the distance of voids between the

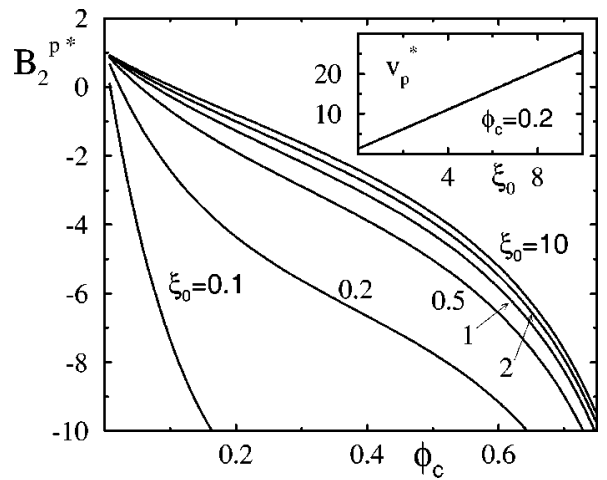

FIG. 9. Normalized polymer second virial coefficient $B_{2}^{P *}$ $=B_{2}^{p} /\left(4 \pi \xi_{0}^{3}\right)$ as a function of the colloid packing fraction for various size ratios $\xi_{0}$ as labeled. The inset shows the normalized polymer intermolecular excluded volume parameter $v_{p}^{*}$ $=v_{p}^{\text {excl }} \xi_{0} /\left(8 \pi l_{p}^{4}\right)$ as a function of the polymer size $\xi_{0}$ and the (indistinguishable) approximation Eq. (45); for the inset, the colloid packing fraction is $\phi_{c}=0.2$.

particles. For larger polymers, this segmental layering is smeared out and after an initial rise $g_{p p}(r)$ monotonically decays to its random value. In the limit where the particles are much smaller than the two polymeric molecules, but of course still much larger than the segmental repeat unit size, the two polymers entwine strongly. For $\xi_{0} \gg 1$ and $\varphi_{p} \rightarrow 0$,

$$
g_{p p}(r) \rightarrow 1+\left[f\left(\phi_{c}\right)-1\right] e^{-r / \xi_{0}},
$$

where

$$
f\left(\phi_{c}\right)=\frac{\phi_{c}\left(6 \lambda_{1}+1-4 \phi_{c}\right)}{2\left(1-\phi_{c}\right)\left(1+2 \phi_{c}\right)}
$$

follows from Eqs. (21) and (25). Within the coil radius, for $r \leqslant \xi_{0}$, the distribution of segments from two chains is almost constant and does not exhibit the self-similar power-law behavior, $1 / r$, of open (Gaussian) fractals. Only for distances larger than the coil size, $r>\xi_{0}$, does the intermolecular segment distribution decay to uncorrelated packing. The probability of segments of different polymer chains to be close, as measured by the pair correlation function contact value $f_{\infty}\left(\phi_{c}\right)$, is determined by the particle packing fraction, and for $\phi_{c} \geqslant 0.11$ becomes (much) higher than the bulk density. This increase is a precursor of the demixing transition at finite polymer concentrations [35]. For $\phi_{c} \approx 0.5$, the density of segments from other chains within a $R_{g}$ distance of a tagged segment is more than an order of magnitude greater than in the absence of colloids. This may have significant consequences for intermolecular processes such as chemical reactions or energy transfer between polymers added at dilute levels to colloidal suspensions or porous materials.

The intermolecular excluded volume parameter, defined as $v_{p}^{\text {excl }}\left(\phi_{c}\right)=-\hat{c}_{p p}(0)$, or the related polymer molecule second virial coefficient $B_{2}^{p}$ determine the importance of the excluded volume interaction on the polymer packing on local and macromolecular distances, respectively. Results are shown in Fig. 9. On local distances, it appears plausible that 
a polymer chain should experience the identical steric repulsion from a segment of its own backbone as from another of the chemically identical macromolecules. Thus, the result in Eq. (26) can be used to discuss the effective excluded volume parameter induced by the depletant particles. For vanishing colloid concentration, where $v_{p}^{\text {excl }}$ and $B_{2}^{p}$ are directly related, the interpretation of polymer chains as repulsive spheres is recovered in PRISM because $v_{p}^{\text {excl }}\left(\phi_{c}\right) \propto 1 / R_{g}$ and therefore $B_{2}^{p} \propto R_{g}^{3}$. Note, that our present model thus does not describe $\Theta$ solvents, where $B_{2}^{p}=0$, even though a Gaussian intramolecular structure was assumed a priori, but rather describes polymers in athermal solutions with the technical simplification to treat the polymer chains as random walks. For results with self-avoiding statistics, see, e.g., Refs. $[48,56]$. The result in Eq. (26), which to a very good approximation simplifies to

$$
v_{p}^{\mathrm{excl}}\left(\phi_{c}\right)=\frac{8 \pi l_{p}^{4}}{\xi_{0}}\left(\frac{1}{1-\phi_{c}}+\frac{3\left(2+\lambda_{1}\right)^{2}}{\lambda_{1}^{2}} \frac{\phi_{c} \xi_{0} / \sigma_{c}}{\left(1-\phi_{c}\right)^{2}}\right),
$$

indicates that the effective local segmental interaction stays repulsive for all size ratios and particle fluid concentrations. Asymptotically for small depletant particles, it becomes independent of the macromolecular size and saturates to a positive value that increases with $\phi_{c}$. This is found even though, mesoscopically, two polymers are induced to intertwine, and for somewhat higher polymer concentration, phase separation into a polymer gas and polymer fluid phase sets in [35]. At macromolecular distances, the effective polymer-polymer molecule interaction can become very attractive if the particle density is high or the colloidal particles are large,

$$
B_{2}^{p} \rightarrow \begin{cases}-\frac{\pi \sigma_{c}^{3}}{12}\left[\frac{\left(1-\phi_{c}\right)^{2} \phi_{c}}{\left(1+2 \phi_{c}\right)^{2}}+\frac{24 \phi_{c}\left(1-\phi_{c}\right)}{\left(1+2 \phi_{c}\right)^{2}} \frac{\xi_{0}}{\sigma_{c}}\right] & \text { for } \xi_{0} \rightarrow 0 \\ \frac{4 \pi \xi_{0}^{3}}{\left(1+2 \phi_{c}\right)}\left[1-\frac{36 \phi_{c}}{\left(1-\phi_{c}\right)} \frac{1+\lambda_{1}+\frac{1}{3} \lambda_{1}^{2}}{\lambda_{1}^{3}}\right] & \text { for } \xi_{0} \rightarrow \infty .\end{cases}
$$

For small polymers, the result known from PY theory for hard sphere mixtures is recovered. It is negative and describes the tendency of the polymer point particles to cluster. Increasing the polymer size somewhat increases this tendency. For large polymers, however, a finite colloid density is required in order for the colloid induced attraction to overwhelm the segmental repulsion. The virial coeficient becomes negative only for $\phi \geqslant 0.108 \ldots$ for $\xi_{0} \gg 1$.

\section{CONCLUSIONS}

We have studied athermal colloid and polymer mixtures and considered the dilute limit of one species using a binarymixture approach, which treats the hard spheres and Gaussian polymer coils on an equal footing. The macromolecular liquid state theory uniquely addresses the structural correlations over the wide range of length scales from the polymer repeat unit size to the molecular sizes. Packing of hard spheres is handled using the reliable Percus-Yevick approximation. Polymer specific effects are captured, which arise from the ability of the polymer coils to deform close to and around the particles. Also, the appropriate polymer correlation length appears in the description because the formation of a polymer mesh is captured. The polymer specific effects become important as soon as the size of the coils is not negligible relative to the colloid radius.

The comparison of the $m$-PY approximation with rigorous field-theoretic results in the dilute colloid limit presented in Sec. V serves to validate our approach, which offers the unique possibility of extension to higher densities. Interesting predictions arise for the colloid induced pair interaction of dilute polymers, which are much larger than the particles. Strong interpenetration is predicted (see Fig. 8) finally leading to fluid-fluid phase separation [35].

In this context it is important to stress that the approximation of Gaussian single-chain correlations is done for purely technical reasons in order to achieve analytical results. Of course this entails that the majority of the scaling predictions derived in the present and previous work [35] bears the wrong exponents if applied to polymers in a good solvent. Intramolecular excluded volume, thermal attractions, and a self-consistent determination of $\omega(q)$ will be included in future numerical studies, and in a number of cases the corrected scaling predictions have already been pointed out $[35,48,56]$.

Colloidal dispersions containing added free polymer are often described by integrating out the polymer degrees of freedom in order to derive an effective colloidal Hamiltonian [7]. However, for interacting polymers there exists no small parameter, and the induced many-particle interactions among the colloids do not generally terminate at a pairwise description, and the polymer density dependence of the parameters in the effective interactions cannot be neglected. Both assumptions enter the description of polymer-colloid mixtures using effective pair potentials like the Asakura-Osawa model $[1,4]$, and are justified only for low concentrations of polymers much smaller than the colloid particles, and preferrably dissolved in $\Theta$ solvents that mimimize the excluded volume 
interaction. The discussion of semidilute polymer solutions in Sec. V clearly identifies the importance of the density dependent polymer blob size or mesh width, which also appears in $\Theta$ solvents [44]. The importance of induced manybody interactions, which naturally are contained in the presented binary mixture approach, was discussed in Refs. $[6,35]$. Treatments of binary mixtures of spheres have been used to describe the colloid-polymer mixtures, replacing the polymer coils by spheres of equal or similar size and neglecting their direct interactions ("phantom sphere models") $[5,8]$. These capture induced many-body interactions but neglect the deformability of polymers around particles and the crossover of the relevant polymer length scale from the coil size to the blob size when reaching semidilute concentrations. Whereas these effects had been well appreciated when adding dilute particles to polymer solutions, their study in concentrated particle solutions in the present approach clearly reveals their importance whenever the size of the polymer coils is not negligible. Their description in recent "soft-colloid" approches to polymers is yet unclear [57,58]. An interesting quantity in this context is the free energy cost for adding polymers to hard sphere fluids because this is the central input for the widely used free volume theory of Lekkerkerker et al. [5]. As Fig. 7 shows polymer deformability leads to strong deviations compared to results based on replacing polymer coils by hard spheres.

A central ingredient of the present approach consists in enforcing thermodynamic consistency for the polymer insertion chemical potential $\left.\delta \mu_{p}\right|_{\varrho_{p}=0}$. The length scale $\lambda$ over which polymer segments are allowed to rearrange close to the particles is determined from equating expressions for $\delta \mu_{p}$ from long wavelength and from local packing information. Although the latter compares favorably with exact results for dilute systems, quantitatively we consider the former coarse-grained approach ("compressibility route") to be more reliable, and also preferentially use it for other thermodynamic quantities within the present approach. Our reasoning rests on three observations.

(i) The used scaling law approach (thread model) to semidilute polymer solutions is coarse grained a priori, so a zero wave vector thermodynamic route is natural. Recently it has been connected to a self-consistent Gaussian field theory [59].

(ii) The compressibility route leads to thermodynamic results far less dependent on the specific closure as can be seen by comparing the present $m$-PY with previous PY results $[20,40,41]$.

(iii) In a microscopic calculation the universality, in fieldtheoretic sense, of the long wavelength structure could be shown explicitly [47], in contrast with the local structure route where microscopic parameters remained as prefactors. In the present context this implies that a full PRISM calculation could lead to a local matching length in Eq. (29) dependent on the excluded volume size. Thermodynamic consistency, nevertheless, should prove a useful concept also for the description of other systems, like polyelectrolyte mixtures [52], where PRISM-based numerical approaches have already given interesting results.

\section{ACKNOWLEDGMENTS}

We gratefully acknowledge fruitful discussions with $\mathrm{L}$. Belloni, S. Egelhaaf, E. Eisenriegler, M. Schmidt, and R. Sear. M.F. was supported by the Deutsche Forschungsgemeinschaft under Grant No. Fu 309/3 and through the SFB 563. K.S.S. was supported by the U.S. DOE Division of Materials Science Grant No. DEFG02-96ER45539 through the UIUC Materials Research Laboratory.

\section{APPENDIX A: FACTORIZATION OF THE INTEGRAL EQUATIONS}

Three-dimensional Fourier transformations leading to $\hat{f}(q)$ of functions $f(r)$ depending on the radius $r$ only shall be simplified to one-dimensional ones as follows:

$$
\begin{gathered}
\hat{f}(q)=2 \pi \int_{-\infty}^{\infty} d r e^{i q r} \tilde{f}(r), \quad \text { where } \\
\tilde{f}(r)=\int_{|r|}^{\infty} d s s f(s) .
\end{gathered}
$$

\section{The limit $\phi_{c} \rightarrow 0$}

Inserting Eq. (12), the first equation of Eqs. (14) becomes

$$
\hat{h}_{12}=\xi^{2} \frac{\hat{\bar{c}}_{12}^{s}}{\left(1+\lambda^{2} q^{2}\right)\left(1+\xi^{2} q^{2}\right)},
$$

which suggests an ansatz for $\bar{c}^{s}$ of the form

$$
\hat{\bar{c}}_{12}^{s}(q)=2 \pi e^{-i q / 2}\left(u_{a}+i \lambda q v_{a}\right)+(1+i q \lambda)(1+i q \xi) \hat{q}_{12}(q) .
$$

This shifts the problem of finding $\bar{c}_{12}^{s}(r)$ for $-\frac{1}{2} \leqslant r \leqslant \frac{1}{2}$ to the problem of finding the (Wiener-Hopf factor) function $q_{12}(r)$, where

$$
\hat{q}_{12}(q)=2 \pi \int_{-1 / 2}^{1 / 2} d r e^{i q r} q_{12}(r),
$$

and $q_{12}(r)=0$ elsewhere. From the required symmetry, $\hat{\bar{c}}_{12}^{s}(q)=\hat{\bar{c}}_{12}^{s}(-q)$, and Eq. (A3), follows the continuity of $q_{12}$ at the upper boundary,

$$
q_{12}\left(\frac{1}{2}\right)=0 .
$$

Inserting Eq. (A3) into Eq. (A2) leads to

$$
\begin{gathered}
(1-i q \lambda)(1-i q \xi) \hat{h}_{12}(q)-\xi^{2} \hat{q}_{12}(q) \\
=\frac{2 \pi \xi^{2} e^{-i q / 2}\left(u_{a}+i \lambda q v_{a}\right)}{(1+i q \lambda)(1+i q \xi)},
\end{gathered}
$$

where by simple inspection the right-hand side has no pole in the lower complex $q$ plane, $\Im q<0$. Thus for $r>-\frac{1}{2}$ the 
Fourier-back transform of Eq. (A6), remembering Eq. (A1), can be performed and leads to

$$
\left(1+\lambda \partial_{r}\right)\left(1+\xi \partial_{r}\right) \tilde{h}_{12}(r)=\xi^{2} q_{12}(r) \quad \text { for } r>-\frac{1}{2} .
$$

For $r \geqslant \frac{1}{2}$, this leads to Eq. (15) and in the overlap region, the excluded volume condition gives

$$
r+\lambda+\xi=\xi^{2} q_{12}^{\prime}(r) \quad \text { for }-\frac{1}{2}<r<\frac{1}{2},
$$

which can easily be integrated using Eq. (A5) and provides the initial condition for the derivatives of $f_{12}$ at $r=\frac{1}{2}$ mentioned in the main text. Also the zero wave vector value of the direct correlation function in Eq. (16) follows from Eq.

(A3) and the symmetry requirement on $\hat{\bar{c}}^{s}$, which fix

$$
u_{a}=-\frac{\xi^{2}+2 \lambda \xi(1+\xi)+\lambda^{2}(1+2 \xi)}{\xi^{2}}, \quad v_{a}=-\frac{\xi+\lambda}{\xi} .
$$

Inserting the ansatz (A3) at $-q$ into the second equation of Eq. (14) leads to

$$
\begin{aligned}
\hat{h}_{22}-\hat{c}_{22}= & \frac{\hat{h}_{12}(q) \varphi_{p}}{2 \pi \xi_{0}}\left(\frac{2 \pi e^{i q / 2}\left(u_{a}-i \lambda q v_{a}\right)}{1+\lambda^{2} q^{2}}\right. \\
& \left.+\frac{(1-i q \xi) \hat{q}_{12}(-q)}{(1+i q \lambda)}\right),
\end{aligned}
$$

where Eq. (A6) shows that the last term on the rhs has no pole for $\Im q<0$ except for a single pole at $q=-i / \lambda$. Introducing the constants

$$
z=\frac{\lambda\left(u_{a}+v_{a}\right)}{2(\lambda+\xi)}, \quad u=z-u_{a}, \quad \text { and } v=-\frac{\xi}{\lambda} z
$$

also the first term on the rhs of Eq. (A10) can be decomposed into poles and zeros in different half planes. On the left-hand side of Eq. (A10), as $c_{22}(r)$ vanishes for $r>1$, its factorization can be achieved using a constant $w$ and an undetermined function $q_{22}(r)$, which vanishes outside the range $0 \leqslant r \leqslant 1$, and whose Fourier transform is given by

$$
\hat{q}_{22}(q)=2 \pi \int_{0}^{1} d r e^{i q r} q_{22}(r) .
$$

Respecting that $\hat{c}_{22}$ is a symmetric function of $q$, the following ansatz is required:

$$
\begin{aligned}
\hat{c}_{22}(q)= & \frac{2 \pi w}{1+\lambda^{2} q^{2}}+\frac{\hat{q}_{22}(q)}{1-i \lambda q}+\frac{\hat{q}_{22}(-q)}{1+i \lambda q}-\frac{\varphi_{p} \xi^{2}}{2 \pi \xi_{0}} \\
& \times \frac{\left[\hat{q}_{12}(q)+2 \pi z e^{-i q / 2}\right]\left[\hat{q}_{12}(-q)+2 \pi z e^{i q / 2}\right]}{1+\lambda^{2} q^{2}}
\end{aligned}
$$

where the requirement of absence of a term of the form $1 / q^{2}$ for $q \rightarrow \infty$, which would correspond to a divergence $c_{22}(r$ $\rightarrow 0$ ) $\sim 1 / r$, determines the constant $w$ from the value of $q_{22}$ at $r=0$,

$$
\frac{w}{2 \lambda}=q_{22}(0)+\frac{\varphi_{p} \xi^{2}}{2 \lambda \xi_{0}} z^{2}
$$

The ansatz Eq. (A13) is useful because it cancels a number of the poles in the lower complex $q$ half plane in Eq. (A10). The required short range of the direct correlation function, $c_{22}(r>1)=0$, however, at first sight is violated by Eq. (A13), which appears to indicate an exponential tail, $r c_{22}(r)=$ const $\times e^{-|r| / \lambda}$ for $|r|>1$. Requiring its prefactor to be zero, i.e., requiring the residues of the rhs of Eq. (A13) at $q= \pm i / \lambda$ to vanish, fixes the initial value of the factor function,

$$
\begin{aligned}
q_{22}(0)= & \frac{-1}{\lambda} \int_{0}^{1} d r e^{r / \lambda} q_{22}(r) \\
& +\frac{\varphi_{p} \xi^{2}}{8 \pi^{2} \lambda \xi_{0}}\left[\hat{q}_{12}(-i / \lambda) \hat{q}_{12}(i / \lambda)\right. \\
& \left.+2 \pi z e^{1 /(2 \lambda)} \hat{q}_{12}(-i / \lambda)+2 \pi z e^{-1 /(2 \lambda)} \hat{q}_{12}(i / \lambda)\right],
\end{aligned}
$$

which is a linear equation in $q_{22}(0)$. Unfortunately, the full expression for $q_{22}(0)$ turns out rather complicated and only simplifies if the fact that $\lambda \ll 1$ holds is recalled in order to neglect corrections of the order $O\left(e^{-1 / \lambda}\right)$.

The factorization of $\hat{c}_{22}$ expressed in Eq. (A13) simplifies the Fourier-back transformation of Eq. (A10), as the poles in the lower complex $q$ plane were identified with Eqs. (A11) and (A13). Thus for $r>0$ one finds after Fourier-back transforming that

$$
\left(1+\lambda \partial_{r}\right) \widetilde{h}_{22}(r)+\frac{\varphi_{p}}{\xi_{0}}\left(u+v \lambda \partial_{r}\right) \widetilde{h}_{12}\left(r-\frac{1}{2}\right)=q_{22}(r)
$$

holds, which proves Eq. (17) and, together with Eq. (A15), also proves Eq. (18) because $g_{22}(1)=q_{22}(1) / \lambda$. In the overlap region, it leads to

$$
q_{22}^{\prime}(r)=r+\lambda+\frac{\varphi_{p}}{\xi_{0}}\left(u r-\frac{u}{2}+v \lambda\right) \quad \text { for } 0<r<1 .
$$


The changes in the contact value upon adding more colloidal particles, i.e., $\left.\left(\partial g_{c p} / \partial \varrho_{c}\right)\right|_{\varrho_{c}=0}$, can be determined in an explicit form from the Wiener-Hopf factorization of the full nonlinear equations [60].

\section{The limit $\varphi_{p} \rightarrow 0$}

For $\varphi_{p} \rightarrow 0$, the colloid structure factor agrees with the PY solution for hard spheres, which can be written in terms of Baxter's factorization function as [42]

$$
\begin{aligned}
& \hat{S}_{22}^{-1}= {\left[1-\hat{q}_{22}(-q)\right]\left[1-\hat{q}_{22}(q)\right], \quad \text { where } } \\
& \hat{q}_{22}(q)=2 \pi \int_{0}^{1} d r q_{22}(r) e^{i q r} \\
&=2 \pi \int_{0}^{1} d r\left(\frac{A}{2}\left(r^{2}-1\right)+B(r-1)\right) e^{i q r}
\end{aligned}
$$

with the coefficients $A=\varrho_{2}\left[\left(1+2 \phi_{c}\right) /\left(1-\phi_{c}\right)^{2}\right]$ and $B$ $=\varrho_{2}\left[-3 \phi_{c} /\left(2 /\left(1-\phi_{c}\right)^{2}\right)\right]$. The first equation of Eqs. (20) can be simplified with the ansatz

$$
\begin{aligned}
\hat{\bar{c}}_{12}^{s}(q)= & \left(1+i q \xi_{0}\right)(1+i q \lambda) \hat{q}_{12}(q) \\
& +e^{-i q / 2}\left[1-\hat{q}_{22}(q)\right] 2 \pi\left(u_{b}+i q \lambda v_{b}\right),
\end{aligned}
$$

as from Baxter's solution it is known that $\left[1-\hat{q}_{22}(q)\right][1$ $\left.+\varrho_{2} \hat{h}_{22}(q)\right]$ has no poles for $\Im q<0$. The function $q_{12}(r)$ again is assumed to vanish outside the overlap region such that Eq. (A4) holds. The required symmetry of $\hat{c}_{12}(q)$ and the known properties of $q_{22}$ further show that Eq. (A5) holds and that $v_{b}=\xi_{0} q_{12}\left(-\frac{1}{2}\right)$ and $u_{b}=-2 \pi q_{22}(0) \lambda v_{b}+v_{b}(1$ $+2 \lambda+\lambda / \xi_{0}$ ). Inserting Eq. (A19) into Eq. (20) and closing the Fourier integrals in the lower complex $q$ half plane thus leads for $r>-\frac{1}{2}$ to

$$
\begin{aligned}
& \left(1+\xi_{0} \partial_{r}\right)\left(1+\lambda \partial_{r}\right) \widetilde{h}_{12}(r) \\
& \quad=\xi_{0}^{2} q_{12}(r)+12 \phi_{c} \xi_{0}^{2} \int_{-1 / 2}^{1 / 2} d s q_{12}(s) \widetilde{h}_{22}(r-s) .
\end{aligned}
$$

This leads to Eq. (21) for $r>\frac{1}{2}$ as $f_{i j}(r)=-\partial_{r} \widetilde{h}_{i j}(r)$ for $r$ $>0$. Within the overlap region, the equation determining $q_{12}$ follows:

$$
r+\xi_{0}+\lambda=\xi_{0}^{2} q_{12}^{\prime}(r)+12 \phi_{c} \xi_{0}^{2} \int_{-1 / 2}^{1 / 2} d s q_{12}(s)(r-s),
$$

which is solved by the result given in Eq. (23) with parameters

$$
a=\frac{1-\phi_{c}\left(1-6 \lambda-6 \xi_{0}\right)}{\left(1-\phi_{c}\right)^{2} \xi_{0}^{2}}, \quad b=\frac{\lambda+\xi_{0}}{\left(1-\phi_{c}\right) \xi_{0}^{2}} .
$$

From the discontinuity of $q_{12}^{\prime}$ at $r=\frac{1}{2}$, the initial condition (22) results. Solving for the parameters

$$
\begin{gathered}
u_{b}=-\frac{\left(\lambda+\xi_{0}\right)\left\{\xi_{0}-\phi \xi_{0}+\lambda\left[1+2 \xi_{0}-\phi\left(1-4 \xi_{0}\right)\right]\right\}}{(1-\phi)^{2} \xi_{0}^{2}}, \\
v_{b}=\frac{\lambda+\xi_{0}}{-\xi_{0}+\phi \xi_{0}},
\end{gathered}
$$

one can obtain Eq. (24) from Eq. (A19). The second equation of Eqs. (20) can be rewritten upon insertion of Eq. (A19),

$$
\begin{aligned}
\hat{h}_{11}- & \frac{\xi_{0}^{4}}{\left(1+q^{2} \xi_{0}^{2}\right)^{2}} \frac{\hat{c}_{11}}{l_{p}^{4}} \\
= & \frac{6 \phi_{c} \xi_{0}^{2}}{\pi} \frac{\hat{h}_{12}(q)}{(1-i q \lambda)\left(1-i q \xi_{0}\right)} \\
& \times\left(\hat{q}_{12}(q)+\frac{2 \pi e^{-i q / 2}\left[1-\hat{q}_{22}(q)\right]\left(u_{b}+i q \lambda v_{b}\right)}{(1+i q \lambda)\left(1+i q \xi_{0}\right)}\right) .
\end{aligned}
$$

Considering the explicit expression for $\hat{h}_{12}(q)$ from Eqs. (20), (A18) and (A19),

$$
\begin{aligned}
\hat{h}_{12}(q)= & \hat{h}_{12}(-q) \\
= & \frac{\xi_{0}^{2} \hat{q}_{12}(-q)}{\left[1-\hat{q}_{22}(-q)\right]\left[1-\hat{q}_{22}(q)\right](1+i q \lambda)\left(1+i q \xi_{0}\right)} \\
& +\frac{2 \pi \xi_{0}^{2} e^{i q / 2}\left(u_{b}-i q \lambda v_{b}\right)}{\left[1-\hat{q}_{22}(q)\right]\left(1+q^{2} \lambda^{2}\right)\left(1+q^{2} \xi_{0}^{2}\right)},
\end{aligned}
$$

one recognizes that the first term of Eq. (A25) multiplied with $\left[1-\hat{q}_{22}(q)\right]$ contributes no poles in the lower $q$ half plane. Thus the poles for $\Im q<0$, which are the only ones contributing for $r>0$, from the second part of Eq. (A24) can be identified and characterized with parameters

$$
\begin{gathered}
z_{\lambda}=\frac{3 \phi_{c} \xi_{0}^{4} \lambda}{\left(\lambda-\xi_{0}\right)\left(\lambda+\xi_{0}\right)^{2}}\left(u_{b}^{2}-v_{b}^{2}\right), \\
\bar{z}_{\xi_{0}}=\frac{3 \phi_{c} \xi_{0}^{3}}{\left(\lambda-\xi_{0}\right)\left(\lambda+\xi_{0}\right)^{2}}\left(\lambda^{2} v_{b}^{2}-u_{b}^{2} \xi_{0}^{2}\right),
\end{gathered}
$$

and $z_{\xi_{0}}=\bar{z}_{\xi_{0}}-\hat{c}_{11} \xi_{0}\left(\lambda-\xi_{0}\right) / 8 \pi l_{p}^{4}$. From Eq. (A24), then, Eq. (25) follows by transformation and differentiation. The last unknown quantity, $\hat{c}_{11}(q=0)$, can be obtained from Eq. (A24) because the rhs vanishes as $\cos ^{2}(q / 2) / q^{8}$ for $q \rightarrow \infty$, as can be seen from Eqs. (20) and (A20). Therefore, the discontinuity of the third derivative at $r=0$ of the transform of $\omega_{q}^{2} \hat{c}_{11}$ needs to be balanced by $\hat{c}_{11}$, 


$$
\left.\partial_{r}^{3} \widetilde{h}_{11}(r)\right|_{r=0}=-\left.\partial_{r}^{2} f_{11}(r)\right|_{r=0}=\frac{\hat{c}_{11}(0)}{4 \pi l_{p}^{4}},
$$

which together with Eq. (25) fixes the polymer excluded volume parameter as given in Eq. (26).

The change in the colloid-contact value upon adding polymers, $\left.\left(\partial g_{c c} / \partial \varrho_{p}\right)\right|_{\varrho_{p}=0}$, can be obtained from the equations at finite density and leads to a rather unwieldy expression [60]. For this work, only its limits are required: $\left.\left(\partial g_{c c} / \partial \varrho_{p}\right)\right|_{\varrho_{p}=0}=O\left(\xi_{0}^{2}\right)$ for small $\xi_{0}$, and when terms of $O\left(\lambda^{2}\right)$ are neglected

$$
\left.\frac{\partial g_{c c}(1)}{\partial \varphi_{p}}\right|_{\varrho_{p}=0} \rightarrow \frac{1 /\left(2 \xi_{0}\right)}{\left(1-\phi_{c}\right)^{2}}+\frac{2 \lambda}{\xi_{0}} \frac{1+2 \phi_{c}}{\left(1-\phi_{c}\right)^{3}} \quad \text { for } \xi_{0} \rightarrow \infty .
$$

\section{APPENDIX B: STEPS IN THE DETERMINATION OF $\boldsymbol{\lambda}$}

In the limit $\xi_{0} \gg 1$ one can use the physically motivated expectation that $\lambda$ becomes small compared to $\xi_{0}$ and is of the order of the colloid diameter. With the ansatz $\lambda$ $=\Lambda\left(\phi_{c}\right)$ for $\xi_{0} \rightarrow \infty$, the expressions in Eqs. (22) and (24) simplify. With Eq. (A28), which entails a further expansion in $\Lambda<1$ up to linear order, the contact route expression Eq. (10) becomes

$$
\begin{aligned}
\left.N \beta \delta \mu_{p}^{(\mathrm{g})}\right|_{\varrho_{p}=0} \rightarrow & 2 \xi_{0}^{2} \phi_{c}^{2 / 3} \int_{0}^{\phi_{c}} d x \frac{1+2 x}{\Lambda(x) x^{2 / 3}(1-x)^{2}} \\
& +24 \xi_{0}^{2} \phi_{c}^{2 / 3} \int_{0}^{\phi_{c}} d x x^{1 / 3}\left[\frac{1 / 2}{(1-x)^{2}}\right. \\
& \left.+2\left(\frac{x}{\phi_{c}}\right)^{1 / 3} \Lambda(x) \frac{1+2 x}{(1-x)^{3}}\right] .
\end{aligned}
$$

By multiplication with powers of $\phi_{c}^{1 / 3}$ and differentiation, this expression can easily be turned into an ordinary differential equation for $\Lambda\left(\phi_{c}\right)$, whose asymptotic solutions are given in Eq. (30).

\section{APPENDIX C: COMPARISON OF $B_{2}^{c}$ FROM OTHER CLOSURES}

The failure of thermodynamic consistency in the colloid second virial coefficient $B_{2}^{c}$ for situations where the effective colloid pair potential is strong warrants a comparison with results obtained with other closures. Because of previous polymer-colloid mixture work in Refs. [40,41] we consider two alternative approximations: the PY closure for the

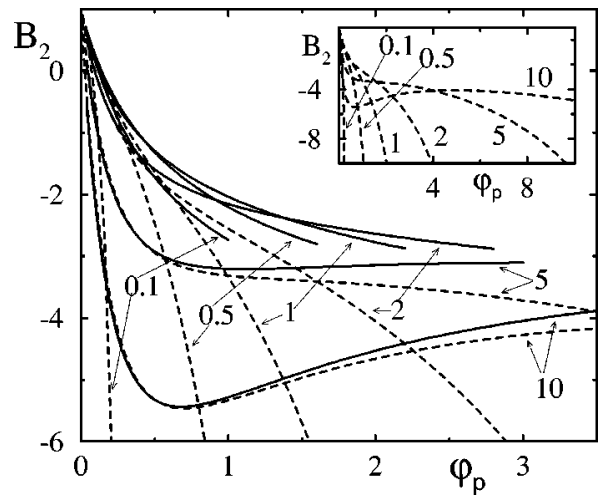

FIG. 10. Colloid second virial coefficient $B_{2}^{c}$ in units of $2 \pi / 3$ as a function of the polymer concentration for various size ratios $\xi_{0}$ as labeled. The solid lines are the compressibility results from Fig. 5 whereas the dashed lines give the corresponding $\mathrm{HNC}$ results. The inset presents the latter in a larger window, showing their drop to large negative values for polymer concentrations far above the overlap one.

colloid-polymer interaction, which corresponds to $\lambda=0$, and the HNC closure [61] or for the colloid correlations. Study of the latter is motivated by the known deficiencies of the PY closure for mixtures of hard spheres, which are (partially) corrected by the HNC closure [61].

The result for the colloid pair correlation function using the HNC closure can easily be obtained from the $m$-PY result via [40]

$$
g_{c c}^{\mathrm{HNC}}(r)=e^{g_{c c}^{m-\mathrm{PY}}}(r)-1 \quad \text { for } r>\sigma_{c} .
$$

Because $g_{c c}^{m-\mathrm{PY}}(r)-1$ and the connected quantity $\beta W(q)$ $=\varrho_{p} c_{c p}^{2}(q) S_{p p}(q)$ in Eq. (14) can be identified as (Fourier transform of) the polymer induced potential [62], the HNC closure agrees with the exact virial expression for colloidal spheres interacting with this (effective) pair potential and the $\sigma_{c} / R_{g} \rightarrow \infty$ blob scaling mean-field results [30]. From Fig. 10 one deduces that, as discussed in Sec. V, the $m$-PY results are not reliable for $\sigma_{c} \gg \xi$ (i.e., small size ratios $\xi_{0}$ or higher polymer concentrations) because the linearization in Eq. (C1) underestimates the strong attraction in these cases. For larger polymers, however, in agreement with the consideration of thermodynamic consistency in Sec. V, the PY linearization of Eq. (C1) is qualitatively and even quantitatively appropriate. The minimum in $B_{2}^{c}$ at the overlap concentration, as well as the semidilute scaling law, are present in the HNC (and $\lambda=0$, PY [40]) results also. The closer quantitative agreement of the HNC results for $B_{2}^{c}$ with the compressibility-route results within $m$-PY is one of the prime reasons we favor this route Eq. (19), over the virialtype route, Eq. (41).
[1] S. Asakura and F. Oosawa, J. Chem. Phys. 22, 1255 (1954); J. Polym. Sci. 33, 183 (1958).

[2] A. Vrij, Pure Appl. Chem. 48, 471 (1976).

[3] J. Traube, Gummi-Ztg. 39, 434 (1925).
[4] A.P. Gast, C.K. Hall, and W.B. Russel, J. Colloid Interface Sci. 96, 251 (1983).

[5] H.N.W. Lekkerkerker, W.C.K. Poon, P.N. Pusey, A. Stroobants, and P.B. Warren, Europhys. Lett. 20, 559 (1992). 
[6] E.J. Meijer and D. Frenkel, Phys. Rev. Lett. 67, 1110 (1991); J. Chem. Phys. 100, 6873 (1994); Physica A 213, 130 (1995).

[7] M. Dijkstra, J.M. Brader, and R. Evans, J. Phys.: Condens. Matter 11, 10079 (1999); M. Dijkstra, R. van Roij, and R. Evans, Phys. Rev. E 59, 5744 (1999).

[8] A.A. Louis, R. Finken, and J.P. Hansen, Europhys. Lett. 46, 741 (1999).

[9] M. Schmidt, H. Löwen, J.M. Brader, and R. Evans, Phys. Rev. Lett. 85, 1934 (2000).

[10] J.M. Brader and R. Evans, Europhys. Lett. 49, 678 (2000).

[11] P.B. Warren, S.M. Ilett, and W.C.K. Poon, Phys. Rev. E 52, 5205 (1995).

[12] D. Rudhardt, C. Bechinger, and P. Leiderer, Phys. Rev. Lett. 81, 1330 (1998).

[13] R. Verma, J.C. Crocker, T.C. Lubensky, and A.G. Yodh, Phys. Rev. Lett. 81, 4004 (1998); Macromolecules 33, 177 (2000).

[14] F.L. Calderon, J. Bibette, and J. Biais, Europhys. Lett. 23, 653 (1993).

[15] S.M. Ilett, A. Orrock, W.C.K. Poon, and P.N. Pusey, Phys. Rev. E 51, 1344 (1995).

[16] R. Tuinier, E. ten Grotenhuis, C. Holt, P.A. Timmins, and C.G. de Kruif, Phys. Rev. E 60, 848 (1999); R. Tuinier and C.G. de Kruif, J. Colloid Interface Sci. 218, 201 (1999); J. Chem. Phys. 110, 9296 (1999).

[17] X. Ye, T. Narayanan, P. Tong, and J.S. Huang, Phys. Rev. Lett. 76, 4640 (1996); X. Ye, T. Narayanan, P. Tong, J.S. Huang, M.Y. Lin, B.L. Carvalho, and L.J. Fetters, Phys. Rev. E 54, 6500 (1996).

[18] A. Moussaid, W.C.K. Poon, P.N. Pusey, and M.F. Soliva, Phys. Rev. Lett. 82, 225 (1999).

[19] R. Tuinier, J.K.G. Dhont, and C.G. de Kruif, Langmuir 16, 1497 (2000); K.-D. Hörner, M. Töpper, and M. Ballauf, ibid. 13, 551 (1997); W. Weiss, K.-D. Hörner, and M. Ballauf, J. Colloid Interface Sci. 213, 417 (1999).

[20] A.M. Kulkarni, A.P. Chatterjee, K.S. Schweizer, and C.F. Zukoski, Phys. Rev. Lett. 83, 4554 (1999); J. Chem. Phys. 113, 9863 (2000).

[21] I. Bodnár and W.D. Oosterbaan, J. Chem. Phys. 106, 7777 (1997).

[22] J. Dzubiella, A. Jusufi, C.N. Likos, C. von Ferber, H. Löwen, J. Stellbrink, J. Allgaier, D. Richter, A.B. Schofield, P.A. Smith, W.C.K. Poon, and P.N. Pusey, Phys. Rev. E 64, 010401(R) (2001).

[23] N.A.M. Verhaegh, J.S. van Duijneveldt, J.K.G. Dhont, and H.N.W. Lekkerkerker, Physica A 230, 409 (1996).

[24] I.D. Robb, P.A. Williams, P. Warren, and R. Tanaka, J. Chem. Soc., Faraday Trans. 91, 3901 (1995).

[25] E. Eisenriegler, A. Hanke, and S. Dietrich, Phys. Rev. E 54, 1134 (1996).

[26] E. Eisenriegler, Phys. Rev. E 55, 3116 (1997).

[27] A. Hanke, E. Eisenriegler, and S. Dietrich, Phys. Rev. E 59, 6853 (1999).

[28] E. Eisenriegler, J. Phys.: Condens. Matter 12, A227 (2000).

[29] E. Eisenriegler, J. Chem. Phys. 113, 5091 (2000).

[30] J.F. Joanny, L. Leibler, and P.G. de Gennes, J. Polym. Sci., Polym. Phys. Ed. 17, 1073 (1979).
[31] P.G. de Gennes, C. R. Acad. Sci. Paris 288B, 359 (1979).

[32] T. Odijk, Macromolecules 29, 1842 (1996); J. Chem. Phys. 106, 3402 (1996); Physica A 278, 347 (2000).

[33] R.P. Sear, Eur. Phys. J. B 1, 313 (1998).

[34] H.M. Schaink and J.A.M. Smit, J. Chem. Phys. 107, 1004 (1997).

[35] M. Fuchs and K.S. Schweizer, Europhys. Lett. 51, 621 (2000).

[36] K.S. Schweizer and J.G. Curro, Phys. Rev. Lett. 58, 246 (1987).

[37] K.S. Schweizer and J.G. Curro, Adv. Polym. Sci. 116, 319 (1994).

[38] K.S. Schweizer and J.G. Curro, Adv. Chem. Phys. 98, 1 (1997).

[39] D. Chandler and H.C. Andersen, J. Chem. Phys. 57, 1930 (1972).

[40] A.P. Chatterjee and K.S. Schweizer, J. Chem. Phys. 109, 10464 (1998); 109, 10477 (1998).

[41] A.P. Chatterjee and K.S. Schweizer, Macromolecules 32, 923 (1999).

[42] J.P. Hansen and I.R. McDonald, Theory of Simple Liquids (Academic Press, London, 1986).

[43] D. Chandler, in Studies in Statistical Mechanics, edited by E.W. Montroll and J.L. Lebowitz (North-Holland, Amsterdam, 1982), Vol. VIII, p. 274.

[44] P.G. de Gennes, Scaling Concepts in Polymer Physics (Cornell University Press, Ithaca, 1979).

[45] K.S. Schweizer and J.G. Curro, Macromolecules 21, 3070 (1988); 21, 3082 (1988).

[46] K.S. Schweizer and J.G. Curro, Chem. Phys. 149, 105 (1990).

[47] M. Fuchs, Z. Phys. B: Condens. Matter 103, 521 (1997).

[48] M. Fuchs and M. Müller, Phys. Rev. E 60, 1921 (1999).

[49] K.S. Schweizer, E.F. David, C. Singh, J.G. Curro, and J.J. Rajasekaran, Macromolecules 28, 1528 (1995).

[50] T.L. Hill, Statistical Mechanics (McGraw-Hill, New York, 1956).

[51] P.G. Khalatur, L.V. Zherenkova, and A.R. Khokhlov, J. Phys. II 7, 543 (1997); N.P. Shusharina, P.G. Khalatur, and A.R. Khokhlov, J. Chem. Phys. 113, 7006 (2000).

[52] P.G. Ferreira, M. Dymitrowska, and L. Belloni, J. Chem. Phys. 113, 9849 (2000).

[53] L. Schäfer, Excluded Volume Effects in Polymer Solutions (Springer, Berlin, 1999).

[54] R. Tuinier, G.A. Vliegenthart, and H.N.W. Lekkerkerker, J. Chem. Phys. 113, 10768 (2000).

[55] Failure to recover the 0.40 exponent is not a failure of PRISM but rather the consequence of the use of a Gaussian $\omega(q)$.

[56] M. Fuchs and K.S. Schweizer, J. Chem. Phys. 106, 347 (1997).

[57] A.A. Louis, P.G. Bolhuis, J.P. Hansen, and E.J. Meijer, Phys. Rev. Lett. 85, 2522 (2000); P.G. Bolhuis, A.A. Louis, J.P. Hansen, and E.J. Meijer, J. Chem. Phys. 114, 4296 (2001).

[58] F. Eurich and P. Maas, J. Chem. Phys. 114, 7655 (2001).

[59] D. Chandler, Phys. Rev. E 48, 2898 (1993).

[60] M. Fuchs and K.S. Schweizer (unpublished).

[61] R. Dickmann, P. Attard, and V. Simonian, J. Chem. Phys. 107, 205 (1997).

[62] C. Grayce and K.S. Schweizer, J. Chem. Phys. 100, 6846 (1994). 